\title{
Review Article \\ New Results on Markov Moment Problem
}

\author{
Octav Olteanu \\ Department of Mathematics-Informatics, University Politehnica of Bucharest, Splaiul Independentei 313, 060042 Bucharest, Romania \\ Correspondence should be addressed to Octav Olteanu; olteanuoctav@yahoo.ie
}

Received 14 September 2012; Revised 17 November 2012; Accepted 19 November 2012

Academic Editor: Remi Léandre

Copyright (C) 2013 Octav Olteanu. This is an open access article distributed under the Creative Commons Attribution License, which permits unrestricted use, distribution, and reproduction in any medium, provided the original work is properly cited.

\begin{abstract}
The present work deals with the existence of the solutions of some Markov moment problems. Necessary conditions, as well as necessary and sufficient conditions, are discussed. One recalls the background containing applications of extension results of linear operators with two constraints to the moment problem and approximation by polynomials on unbounded closed finite-dimensional subsets. Two domain spaces are considered: spaces of absolute integrable functions and spaces of analytic functions. Operator valued moment problems are solved in the latter case. In this paper, there is a section that contains new results, making the connection to some other topics: bang-bang principle, truncated moment problem, weak compactness, and convergence. Finally, a general independent statement with respect to polynomials is discussed.
\end{abstract}

\section{Introduction and Known Results}

We recall that the classical formulation of the moment problem, under the terms of T. Stieltjes, given in 1894-1895 (see [1] for details), finds the repartition of the positive mass on the semiaxis $[0, \infty)$, if the moments of arbitrary orders $k(k=0,1,2, \ldots)$ are given. Precisely, in the Stieltjes moment problem, a sequence of real numbers $\left(s_{k}\right)_{k \geq 0}$ is given, and one looks for a nondecreasing real function $\sigma(t)(t \geq 0)$ that verifies the moment conditions:

$$
\int_{0}^{\infty} t^{k} d \sigma=s_{k} \quad(k=0,1,2, \ldots) .
$$

This is a one-dimensional moment problem, on an unbounded interval. The existence, the uniqueness, and the construction of the solution are considered. The present paper concerns firstly the existence problem. If the interval is replaced by a subset of $R^{n}, n \geq 2$, we have a multidimensional moment problem. If the sequence of the real numbers moments becomes a sequence of operators, we have an operator-valued moment problem. Most of the problems appearing in applications require not only positive, but also an upper constraint on the solution. This is the Markov moment problem.
Applying extension Hahn-Banach type results in existence questions concerning the moment problem is a wellknown technique [1-13]. One of the most useful results is the lemma of the majorizing subspace (see [14, Section 5.1.2] for the proof of the lattice version of this lemma; see also [15]). It says that if $f$ is a linear positive operator on a subspace $S$ of the ordered vector space $X$, the target space being an ordercomplete vector lattice $Y$, and for each $x \in X$, there is $s \in S, x \leq$ $s$, then $f$ has a linear positive extension $F: X \rightarrow Y$. Another geometric remark is that in the real case, the sublinear functional from Hahn-Banach theorem can be replaced by a convex one. The theorem remains valid when the convex dominating functional is defined on a convex subset $A$ with some properties with respect to the subspace $S$ (e.g., $S \cap$ $\operatorname{ri}(A) \neq \Phi)$, where $\operatorname{ri}(A)$ is the relative interior of $A$. The problem is to find necessary and sufficient conditions for the existence of a solution, preserving sandwich conditions. Here we recall an answer published in 1978 [16], without loosing convexity, but strongly generalizing the classical result. The first detailed proof was published in [17]. A proof in terms of the moment problem was published in [12]. Parts of these generalizations of the Hahn-Banach principle are involved in the present work too. Throughout this first part, $X$ will be a real vector space, $Y$ an order-complete vector lattice, $A, B \subset X$ a convex subsets, $q: A \rightarrow Y$ a concave operator, 
$p: B \rightarrow Y$ a convex operator, $S \subset X$ a vector subspace, $f: S \rightarrow Y$ a linear operator.

Theorem 1. Assume that $\left.f\right|_{S \cap A} \geq\left. q\right|_{S \cap A},\left.f\right|_{S \cap B} \leq\left. p\right|_{S \cap B}$. The following assertions are equivalent:

(a) there is a linear extension $F: X \rightarrow Y$ of the operator $f$ such that

$$
\left.F\right|_{A} \geq q,\left.\quad F\right|_{B} \leq p
$$

(b) there are $p_{1}: A \rightarrow Y$ convex and $q_{1}: B \rightarrow Y$ concave operators such that for all

$$
\left(\rho, t, \lambda^{\prime}, a_{1}, a^{\prime}, b_{1}, b^{\prime}, v\right) \in[0,1]^{2} \times(0, \infty) \times A^{2} \times B^{2} \times S,
$$

One has:

$$
\begin{aligned}
(1-t) a_{1}-t b_{1} & =v+\lambda^{\prime}\left[(1-\rho) a^{\prime}-\rho b^{\prime}\right] \\
& \Longrightarrow(1-t) p_{1}\left(a_{1}\right)-t q_{1}\left(b_{1}\right) \\
& \geq f(v)+\lambda^{\prime}\left[(1-\rho) q\left(a^{\prime}\right)-\rho p\left(b^{\prime}\right)\right] .
\end{aligned}
$$

The following result related to the theorem of $\mathrm{H}$. Bauer (see [15, Section 5.4]) is deduced.

Theorem 2. Let $X$ be a preordered vector space with its positive cone $X_{+}, p: X \rightarrow Y$ a convex operator, $S \subset X$ a vector subspace, and $f: S \rightarrow Y$ a linear positive operator. The following assertions are equivalent:

(a) there is a linear positive extension $F: X \rightarrow Y$ off such that

$$
F(x) \leq p(x) \quad \forall x \in X
$$

(b) $f\left(x^{\prime}\right) \leq p(x)$ for all $\left(x^{\prime}, x\right) \in S \times X$ such that $x^{\prime} \leq x$.

Now, we can deduce the main results on the abstract moment problem [11].

Theorem 3. Let $X, Y, p: X \rightarrow Y$ be as in Theorem $2,\left\{x_{j}\right\}_{j \in J} \subset X,\left\{y_{j}\right\}_{j \in J} \subset Y$ given families. The following statements are equivalent:

(a) there is a linear positive operator $F: X \rightarrow Y$ such that

$$
F\left(x_{j}\right)=y_{j} \quad \forall j \in J, \quad F(x) \leq p(x) \quad \forall x \in X ;
$$

(b) for any finite subset $J_{0} \subset J$ and any $\left\{\lambda_{j}\right\}_{j \in J_{0}} \subset R$, one has

$$
\sum_{j \in J_{0}} \lambda_{j} x_{j} \leq x \Longrightarrow \sum_{j \in J_{0}} \lambda_{j} y_{j} \leq p(x)
$$

A clearer sandwich-moment problem variant is the following one.
Theorem 4. Let $X, Y,\left\{x_{j}\right\}_{j \in J},\left\{y_{j}\right\}_{j \in J}$ be as in Theorem 3 and $F_{1}, F_{2} \in L(X, Y)$ two linear operators. The following statements are equivalent:

(a) there is a linear operator $F \in L(X, Y)$ such that

$$
\begin{gathered}
F_{1}(x) \leq F(x) \leq F_{2}(x) \quad \forall x \in X_{+}, \\
F\left(x_{j}\right)=y_{j} \quad \forall j \in J
\end{gathered}
$$

(b) for any finite subset $J_{0} \subset J$ and any $\left\{\lambda_{j}\right\}_{j \in J_{0}} \subset R$, one has:

$$
\begin{gathered}
\left(\sum_{j \in J_{0}} \lambda_{j} x_{j}=\varphi_{2}-\varphi_{1}, \varphi_{1}, \varphi_{2} \in X_{+}\right) \\
\Longrightarrow \sum_{j \in J_{0}} \lambda_{j} y_{j} \leq F_{2}\left(\varphi_{2}\right)-F_{1}\left(\varphi_{1}\right) .
\end{gathered}
$$

Some of the results of this work are applications of the theorems stated previously. Most of the results were published in the last ten years, a few of them being new (Section 4).

For uniqueness of the solution, see [1, 3, 4, 18-22]. Several other interesting related results are contained in [20, 23] (application of fixed-point principle, iterative methods, and complex functions methods) [8,24], (construction of a solution) $[13,25]$. For stable algorithms related to the finite Markov moment problem, see [20, 24]. Similar results are deduced in Section 4 of the present work, by using other methods: bang-bang principle, weak compactness, weak approximation [26], characteristic functions as extreme "points," Gram-Schmidt procedure, and cell-decomposition. These methods seem to work in arbitrary several dimensions.

\section{Approximation on Unbounded Subsets and Moment Problems in Spaces of "Even" Functions}

The results of this section are using those published in [9, 10]. We recall the following recent approximation lemmas concerning the approximation on closed unbounded finitedimensional subsets.

Lemma 5 (see [9] and [10, Lemma 1.3(d)]). If $x \in$ $C_{0}([0, \infty) \times[0, \infty))$ is a nonnegative continuous function with compact support, then there exists a sequence $\left(p_{m}\right)_{m}$ of positive polynomials on $[0, \infty) \times[0, \infty)$, such that

$$
p_{m}(t)>x(t) \quad \forall t \geq 0, \quad \forall m \in Z_{+}, \quad p_{m} \longrightarrow x
$$

uniformly on compact subsets of $[0, \infty) \times[0, \infty)$.

The idea of the proof is to add the $\infty$ point and to apply the Stone-Weierstrass theorem to the subalgebra generated by the functions $\exp \left(-m t_{1}-n t_{2}\right), m, n \in Z_{+}$. Then, one uses 
for each such exp-function suitable majorizing or minorizing partial polynomial sums, as well as the relation

$$
\begin{aligned}
\exp (s) & -\left(1+\frac{s}{1 !}+\frac{s^{2}}{2 !}+\cdots+\frac{s^{m}}{m !}\right) \\
& =\frac{\exp (s)}{m !} \int_{0}^{s} \exp (-t) \cdot t^{m} d t, \quad m \in Z_{+} .
\end{aligned}
$$

Note that [10, Lemma 1.4] asserts the density of positive polynomials in $\left(L_{v}^{1}(A)\right)_{+}$, for any closed subset $A$ of a finitedimensional space, $v$, being a positive regular Borel $\mathrm{M}$ determinate measure. Here is the exact formulation of this result.

Lemma 6 (see [10, Lemma 1.4]). Let $A \subset R^{n}$ be an arbitrary closed subset and $v$ an $M$-determinate positive regular Borel measure on $A$, with finite moments of all natural orders. Then, for any $x \in\left(C_{0}(A)\right)_{+}$such that $x \leq p \in P$ for some polynomial $p$, there exists a sequence $\left(p_{m}\right)_{m}, p_{m} \in P_{+}, p_{m} \geq x, p_{m} \rightarrow x$ in $L_{v}^{1}(A)$. In particular, one has:

$$
\lim \int_{A} p_{m}(t) d v=\int_{A} x(t) d v
$$

$P_{+}$is dense in $\left(L_{v}^{1}(A)\right)_{+}$, and $P$ is dense in $L_{v}^{1}(A)$, on $A$.

Recall that $P$ is the subspace of all polynomial functions

Lemma 7. For any simple function of the form

$$
\begin{gathered}
s=\sum_{j=1}^{N} \alpha_{j} \chi_{I_{j}}, \\
\alpha_{j} \geq 0 \quad j=1, \ldots, N, \quad I_{j} \subset[0, \infty) \quad j=1, \ldots, N,
\end{gathered}
$$

being intervals, there exists a sequence of polynomial

$$
\left(p_{m}\right)_{m}, p_{m} \longrightarrow s \text { in } L_{v}^{1}([0, \infty)), p_{m} \geq s \quad \forall m,
$$

where $v$ is an M-determinate positive regular Borel measure with finite moments of all natural orders. If $s$ is extended to an even function in $R$, then the polynomials $p_{m}, m \in Z_{+}$are restrictions to the positive semiaxes of even polynomials in $R$.

Note that in the preceding Lemmas $5-7$, the sequence $\left(p_{m}\right)_{m}$ is not monotone, but its elements are positive on their domain. The proof of Lemma 6 uses Hahn-Banach theorem, while that of Lemma 5 is more "constructive," the latter being a statement independent with respect to the notion of a measure.

Remark 8. The general form of positive even polynomials on the real axes is

$$
p_{2}(t)=p_{2,1}^{2}\left(t^{2}\right)+t^{2} p_{2,2}^{2}\left(t^{2}\right), \quad t \in R, p_{2, j} \in R[t], j=1,2 .
$$

We start by proving some results on the one-dimensional moment problem.
Theorem 9 (see [10, Theorem 2.1]). Let $v$ be a positive determinate Borel regular measure on $R$ with finite moments $m_{2 k}=\int_{R} t^{2 k} d v\left(t^{2}\right)$ of all orders, $k \in Z_{+}$such that $\int_{R} x_{1}(t) d v=$ 0 for any odd function $x_{1} \in L_{v}^{1}(R)$. Let $\left(y_{2 k}\right)_{k \in Z_{+}}$be a given sequence of real numbers.

The following statements are equivalent:

(a) there exists a Borel function $h$ such that

$$
\begin{gathered}
\int_{R} t^{2 k} h\left(t^{2}\right) d v\left(t^{2}\right)=y_{2 k}, \\
\int_{R} t^{2 k+1} h\left(t^{2}\right) d v=0, \\
\forall k \in Z_{+}, \\
0 \leq h\left(t^{2}\right) \leq 1 \quad d v \text {-a.e. in } R ;
\end{gathered}
$$

(b) for any finite subset $J_{0} \subset Z_{+}$and any $\left\{\lambda_{j} ; j \in J_{0}\right\} \subset R$, one has

$$
\begin{aligned}
& 0 \leq \sum_{i, j \in J_{0}} \lambda_{i} \lambda_{j} y_{2 i+2 j} \leq \sum_{i, j \in J_{0}} \lambda_{i} \lambda_{j} \int_{R} t^{2 i+2 j} d v, \\
& 0 \leq \sum_{i, j \in J_{0}} \lambda_{i} \lambda_{j} y_{2 i+2 j+2} \leq \sum_{i, j \in J_{0}} \lambda_{i} \lambda_{j} \int_{R} t^{2 i+2 j+2} d v .
\end{aligned}
$$

Proof. The hypothesis (a) implies that (b) is obvious because of the qualities of $h$.

(b) $\Rightarrow$ (a). Since $v$ vanishes on the subspace of odd functions, the same property is valid for $h\left(t^{2}\right) \mathrm{d} v$. The problem can be reduced to a corresponding one on the positive semiaxis $u=t^{2} \geq 0$. Using the form of the positive polynomials in $u \in R_{+}[1]$, the hypothesis (b) yields

$$
\begin{gathered}
f\left(\sum_{j \in J_{0}} \lambda_{j} \widetilde{x}_{j}\right):=\sum_{j \in J_{0}} \frac{\lambda_{j} y_{2 j}}{2}, \quad \widetilde{x}_{j}(u)=u^{j}, j \in Z_{+} \\
\Longrightarrow 0 \leq f(p) \leq \int_{0}^{\infty} p(u) d v \quad \forall p \in P_{+} .
\end{gathered}
$$

Since $P$ is a majorizing subspace in

$$
X:=\left\{x \in L_{v}^{1}([0, \infty)) ; \exists p \in P,|x(u)| \leq p(u), \forall u \geq 0\right\},
$$

there exists a positive linear extension $F \in X^{*}$ of $f$. By Haviland-Riesz representation theorem, there exists a representing positive regular Borel measure $\mu$ on $[0, \infty)$ such that

$$
\mu(p)=F(p)=f(p) \leq v(p), \quad p \in P_{+} .
$$

Applying Haviland-Riesz theorem once more, we infer the existence of a positive regular Borel measure $\lambda$ on $[0, \infty)$, such that

$$
\lambda(p)=(\nu-\mu)(p) \Longrightarrow v(p)=\lambda(p)+\mu(p), \quad p \in P .
$$


Since $v$ is $M$-determinate, application of Lemma 7 and the last relation lead to

$$
v(B)=\lambda(B)+\mu(B), \quad \forall B \subset[0, \infty),
$$

Borel subset. In particular, $\mu$ is absolutely continuous with respect to $v$, hence there exists $h_{1} \in L_{v}^{1}([0, \infty))$ such that $d \mu=h_{1} d \nu$ on simple functions. From [28, Theorem 1.40], we infer that $0 \leq h_{1} \leq 1 \quad v$-a.e. Since $v$ was a positive regular Borel measure, we can find a Borel function $h$ such that $h=h_{1} v$-a.e. The previous relations lead to

$$
\begin{aligned}
\int_{0}^{\infty} u^{j} h(u) d v & =\int_{0}^{\infty} u^{j} d \mu=F\left(\widetilde{x}_{j}\right)=\frac{y_{2 j}}{2} \\
& \Longrightarrow \int_{-\infty}^{\infty} t^{2 j} h\left(t^{2}\right) d v\left(t^{2}\right) \\
& =2 \frac{y_{2 j}}{2}=y_{2 j}
\end{aligned}
$$

This concludes the proof.

Corollary 10. Let $\alpha>0$ and $\left\{y_{2 k}\right\}_{k \in Z_{+}} \subset R$. The following statements are equivalent:

(a) there exists a Borel function $h$, such that

$$
\begin{aligned}
& \int_{R} t^{2 k} h\left(t^{2}\right) d t=y_{2 k}, \quad \int_{R} t^{2 k+1} h\left(t^{2}\right) d t=0, \quad \forall k \in Z_{+}, \\
& 0 \leq h\left(t^{2}\right) \leq \frac{1}{\sqrt{2 \pi} \alpha} \exp \left(-\frac{t^{2}}{2 \alpha^{2}}\right) \text { a.e. in } R ;
\end{aligned}
$$

(b) for any finite subset $J_{0} \subset Z_{+}$and any $\left\{\lambda_{j} ; j \in J_{0}\right\} \subset R$, one has

$$
\begin{aligned}
& 0 \leq \sum_{i, j \in J} \lambda_{i} \lambda_{j} y_{2 i+2 j} \leq \sum_{i, j \in J} \lambda_{i} \lambda_{j} \alpha^{2(i+j)}[2(i+j)-1] ! ! \\
& 0 \leq \sum_{i, j \in J} \lambda_{i} \lambda_{j} y_{2 i+2 j+2} \leq \sum_{i, j \in J} \lambda_{i} \lambda_{j} \alpha^{2(i+j+1)}[2(i+j+1)-1] ! !
\end{aligned}
$$

Proof. One applies Theorem 9 to $d v=(1 /(\sqrt{2 \pi} \alpha)) \exp \left(-t^{2} /\right.$ $\left.2 \alpha^{2}\right) d t$, as a measure on $R$.

Due to Carleman criterion, $d v$ is $M$-determinate. A straightforward computation shows that

$$
\int_{R} t^{2 k} d v=\alpha^{2 k}(2 k-1) ! !, \quad k \geq 1, k \in Z_{+} .
$$

The conclusion follows.

Using a quite different proof with respect to that of Theorem 9, we proved the following "abstract operatorial version." Let $v$ be a positive measure as in Theorem 9, vanishing on the subspace of odd functions from $L_{v}^{1}(R)$. Let $Y$ be a Banach lattice, which is order complete. Let $x_{j}(t)=t^{j}$, $t \in R, t^{2}=u \in[0, \infty), \tilde{x}_{j}(u)=u^{j}$. Then, the following theorem holds.
Theorem 11 (see [10, Theorem 2.1]). Let $F_{2}: L_{v}^{1}(R) \rightarrow Y$ be a linear continuous positive operator vanishing on the subspace of odd functions and $\left\{y_{2 k}\right\}_{k} \subset Y$.

The following statements are equivalent:

(a) there exists a linear operator $F: L_{v}^{1}(R) \rightarrow Y$ such that

$$
\begin{gathered}
F\left(x_{2 k}\right)=y_{2 k}, \quad F\left(x_{2 k+1}\right)=0, \quad k \in Z_{+} ; \\
0 \leq F(x) \leq F_{2}(x) \quad \forall x \in\left(L_{v}^{1}(R)\right)_{+} \Longrightarrow\|F\| \leq\left\|F_{2}\right\| ;
\end{gathered}
$$

(b) for any finite subset $J_{0} \subset Z_{+}$and any $\left\{\lambda_{j} ; j \in J_{0}\right\} \subset R$, one has

$$
\begin{gathered}
0 \leq \sum_{i, j \in J_{0}} \lambda_{i} \lambda_{j} y_{2 i+2 j} \leq \sum_{i, j \in J_{0}} \lambda_{i} \lambda_{j} F_{2}\left(x_{2 i+2 j}\right), \\
0 \leq \sum_{i, j \in J_{0}} \lambda_{i} \lambda_{j} y_{2 i+2 j+2} \leq \sum_{i, j \in J_{0}} \lambda_{i} \lambda_{j} F_{2}\left(x_{2 i+2 j+2}\right) .
\end{gathered}
$$

In the sequel, $Y$ will be the order-complete vector lattice, which is also a commutative Banach algebra of selfadjoint operators $[6,14]$. Let $H$ be a Hilbert space $A$ a self-adjoint operator from $H$ into $H$,

$$
\begin{aligned}
& Y_{1}=Y_{1}(A)=\{T \in \mathrm{A}(H) ; A T=T A\}, \\
& Y=\left\{T \in Y_{1} ; T U=U T, \forall U \in Y_{1}\right\} .
\end{aligned}
$$

Let $X$ be the space of all continuous functions $x$ applying $R$ into $R$, such that $|x|$ is majorized by a polynomial on $R$. Denote by $X_{2}$ the subspace of even functions, and $S(A)$ will be the spectrum of $A$.

Theorem 12. Let $\left\{B_{2 k}\right\}_{k \in Z_{+}} \subset Y=Y(A)$. The following statements are equivalent:

(a) there exists $F \in L(X, Y)$ such that

$$
\begin{aligned}
& F\left(x_{2 k}\right)=B_{2 k}, \quad F\left(x_{2 k+1}\right)=0 \quad \forall k \in Z_{+}, \\
& 0 \leq F\left(\varphi_{2} \cdot \chi_{S(A)}\right) \leq \int_{S(A)} \varphi_{2}(t) d E_{A}, \quad \varphi \in X_{2,+}
\end{aligned}
$$

(b) for any finite subset $J_{0} \subset Z_{+}$and any $\left\{\lambda_{j} ; j \in J_{0}\right\} \subset R$, one has

$$
\begin{aligned}
& 0 \leq \sum_{i, j \in J_{0}} \lambda_{i} \lambda_{j} B_{2 i+2 j} \leq \sum_{i, j \in J_{0}} \lambda_{i} \lambda_{j} A^{2 i+2 j}, \\
& 0 \leq \sum_{i, j \in J_{0}} \lambda_{i} \lambda_{j} B_{2 i+2 j+2} \leq \sum_{i, j \in J_{0}} \lambda_{i} \lambda_{j} A^{2 i+2 j+2} .
\end{aligned}
$$

Proof. (b) $\Rightarrow$ (a). Let

$$
f: P \cap X_{2} \rightarrow Y, \quad f\left(\sum_{j \in J_{0}} \lambda_{j} x_{2 j}\right)=\sum_{j \in J_{0}} \lambda_{j} B_{2 j} .
$$


Then, the assumptions of (b) become

$$
0 \leq f\left(p_{2}\right) \leq \int_{S(A)} p_{2}(t) d E_{A}, \quad \forall p_{2} \in P_{2,+} .
$$

Since $P_{2}$ is a majorizing subspace in $X_{2}, f$ has a positive linear extension

$$
F_{2} \in L_{+}\left(X_{2}, Y\right)
$$

Define

$$
\begin{aligned}
& F: X \longrightarrow Y, \quad F(x)=F\left(x_{1}+x_{2}\right)=F_{2}\left(x_{2}\right), \\
& x_{2}(t)=\frac{1}{2}(x(t)+x(-t)), \quad t \in R .
\end{aligned}
$$

Then, $F$ is a linear positive extension of $f$ to the space $X$. The first two properties of the solution $F$ hold. Using Urysohn's lemma, for any $\varphi_{2} \in\left(X_{2}\right)_{+}$, there is a sequence

$$
\left\{\tilde{\varphi}_{2, l}\right\}_{l} \subset\left(C_{c}(R)\right)_{+} \cap X_{2}, \quad \tilde{\varphi}_{2, l} \downarrow \varphi_{2} \cdot \chi_{S(A)} .
$$

Applications of the one-dimensional variant of Lemma 5 lead to the existence of a sequence of polynomial functions $\left(p_{m}\right)_{m}$ such that

$$
\left.p_{m}\right|_{S(A)} \longrightarrow \varphi_{2} \chi_{S(A)} \text { uniformly on } S(A) .
$$

These arguments and the positivity of $F$ yield

$$
\begin{aligned}
0 & \leq F\left(\varphi_{2} \cdot \chi_{S(A)}\right) \\
& \leq \inf F\left(p_{m}\right)=\inf f\left(p_{m}\right) \\
& \leq \inf \int_{S(A)} p_{m}(t) d E_{A}=\int_{S(A)} \varphi_{2}(t) d E_{A} .
\end{aligned}
$$

The proof is finished.

The last result of this section concerns the multidimensional moment problems with solutions vanishing on some subspaces. We prove the result only for the bidimensional case. Let

$$
\begin{gathered}
X=\left\{x: R^{2} \longrightarrow R ; x \text { continuous, } \exists p \in P\right. \\
\text { s.t. } \left.\left|x\left(t_{1}, t_{2}\right)\right| \leq p\left(t_{1}, t_{2}\right), \forall\left(t_{1}, t_{2}\right) \in R^{2}\right\}, \\
X_{2}=\left\{x \in X ; \quad x\left(t_{1}, t_{2}\right)=x\left( \pm t_{1}, \pm t_{2}\right),\left(t_{1}, t_{2}\right) \in R^{2}\right\} .
\end{gathered}
$$

If $H$ is a Hilbert space and $A_{1}, A_{2} \in \mathrm{A}(H)$ are two bounded positive commuting selfadjoint operators, one defines

$$
\begin{aligned}
& Y_{1}=Y_{1}\left(A_{1}, A_{2}\right)=\left\{T \in \mathrm{A}(H) ; T A_{j}=A_{j} T, j=1,2\right\}, \\
& Y=\left\{U \in Y_{1} ; T U=U T, \forall T \in Y_{1}\right\} .
\end{aligned}
$$

Then, $Y$ endowed with the usual order relation on selfadjoint operators is an order-complete vector lattice and a commutative Banach algebra.
Theorem 13. Let $\left\{B_{(2 m, 2 n)}\right\}_{(m, n) \in Z_{+}^{2}} \subset Y\left(A_{1}, A_{2}\right)$ be a given sequence. The following statements are equivalent:

(a) there exists $F \in L(X, Y)$ such that

$$
\begin{aligned}
& F\left(x_{(2 m, 2 n)}\right)=B_{(2 m, 2 n)}, \quad(m, n) \in Z_{+}^{2}, \\
& F\left(x_{(k, l)}\right)=0, \quad \forall(k, l) \in\left(Z_{+}\right)^{2} \backslash\left(2 Z_{+}\right)^{2}, \\
& 0 \leq F\left(x_{2}\right) \\
& \quad \leq \int_{S\left(A_{1}\right) \times S\left(A_{2}\right)} x_{2}\left(t_{1}, t_{2}\right) d E_{A_{1}} d E_{A_{2}} \quad \forall x_{2} \in\left(X_{2}\right)_{+}, \\
& |F(\varphi)| \leq \int_{S\left(A_{1}\right) \times S\left(A_{2}\right)}\left|\varphi\left(t_{1}, t_{2}\right)\right| d E_{A_{1}} d E_{A_{2}} \quad \forall \varphi \in X_{2} ;
\end{aligned}
$$

(b) for all $J_{1}, J_{2} \subset Z_{+}$finite subsets, for all $\left\{a_{j} ; j \in J_{1}\right\}$, and for all $\left\{b_{m} ; m \in J_{2}\right\} \subset R$, one has

$$
\begin{aligned}
0 \leq \sum_{\substack{i, j \in J_{1}, m, n \in J_{2}}} a_{i} a_{j} b_{m} b_{n} B_{(2 i+2 j, 2 m+2 n)} \\
\leq \sum_{\substack{i, j \in J_{1}, m, n \in J_{2}}} a_{i} a_{j} b_{m} b_{n} A_{1}^{2 i+2 j} A_{2}^{2 m+2 n} \\
0 \leq \sum_{\substack{i, j \in J_{1}, m, n \in J_{2}}} a_{i} a_{j} b_{m} b_{n} B_{(2 i+2 j+2,2 m+2 n)} \\
\leq \sum_{\substack{i, j \in J_{1}, m, n \in J_{2}}} a_{i} a_{j} b_{m} b_{n} A_{1}^{2 i+2 j+2} A_{2}^{2 m+2 n}
\end{aligned}
$$$$
0 \leq \sum_{\substack{i, j \in J_{1}, m, n \in J_{2}}} a_{i} a_{j} b_{m} b_{n} B_{(2 i+2 j, 2 m+2 n+2)}
$$$$
\leq \sum_{\substack{i, j \in J_{1}, m, n \in J_{2}}} a_{i} a_{j} b_{m} b_{n} A_{1}^{2 i+2 j} A_{2}^{2 m+2 n+2}
$$

$$
\begin{aligned}
0 & \leq \sum_{\substack{i, j \in J_{1}, m, n \in J_{2}}} a_{i} a_{j} b_{m} b_{n} B_{(2 i+2 j+2,2 m+2 n+2)} \\
& \leq \sum_{\substack{i, j \in J_{1}, m, n \in J_{2}}} a_{i} a_{j} b_{m} b_{n} A_{1}^{2 i+2 j+2} A_{2}^{2 m+2 n+2} .
\end{aligned}
$$

Proof. Since (a) $\Rightarrow$ (b) is obvious, we have to prove the implication (b) $\Rightarrow(\mathrm{a})$. By Stone-Weierstrass theorem, we know that the vector subspace $S$ generated by the functions

$$
\begin{aligned}
& f\left(t_{1}, t_{2}\right)=f_{1}\left(t_{1}\right) \cdot f_{2}\left(t_{2}\right)=\left(f_{1} \otimes f_{2}\right)\left(t_{1}, t_{2}\right), \\
& f_{j} \in C\left(S\left(A_{j}\right)\right), \quad j=1,2,
\end{aligned}
$$

is a dense subalgebra of $C\left(S\left(A_{1}\right) \times S\left(A_{2}\right)\right)$. Hence, for any $x \in\left(C\left(S\left(A_{1}\right) \times S\left(A_{2}\right)\right)\right)_{+}$, there exists a sequence $\left(f_{m}\right)_{m}$ of elements from $S$, which converges uniformly to $x$ on 
$S\left(A_{1}\right) \times S\left(A_{2}\right)$, such that $f_{m}>x \geq 0$ in $S\left(A_{1}\right) \times S\left(A_{2}\right)$. For any function from $C\left(S\left(A_{1}\right) \times S\left(A_{2}\right)\right)$, we consider its extension to $[0, \infty) \times[0, \infty)$ which vanishes outside $S\left(A_{1}\right) \times S\left(A_{2}\right)$, and then we extend again this function to $R^{2}$, such that $\tilde{x}\left(t_{1}, t_{2}\right)=$ $\widetilde{x}\left( \pm t_{1}, \pm t_{2}\right)$, for all $\left(t_{1}, t_{2}\right) \in R^{2}$. If we write

$$
f_{m}\left(t_{1}, t_{2}\right)=f_{m, 1}\left(t_{1}\right) f_{m, 2}\left(t_{2}\right), \quad\left(t_{1}, t_{2}\right) \in R^{2},
$$

where $f_{m, j}$ are extended to $R_{+}$such that to vanish outside $S\left(A_{j}\right), j=1,2$, and to $R$ such that to be even functions, we infer that $f_{m, 1}, f_{m, 2}$ are even bounded measurable nonnegative functions on $R$ with compact support. An application of Luzin's theorem and of the one-dimensional version of Lemma 5 [10, lemma 1.3(b)] leads to uniform approximation of $f_{m, j}$ on $S\left(A_{j}\right), j=1,2$, by even positive polynomials on $R$. Using the representation of these polynomials given in Remark 8 , (b) is rewritten as

$$
\begin{gathered}
f\left(\left(\sum a_{j} t_{1}^{2 j}\right)\left(\sum b_{m} t_{2}^{2 m}\right)\right):=\sum a_{j} b_{m} B_{(2 j, 2 m)} \\
\Longrightarrow 0 \leq f\left(p_{1} p_{2}\right) \\
\leq \int_{S\left(A_{1}\right) \times S\left(A_{2}\right)} p_{1}\left(t_{1}\right) \cdot p_{2}\left(t_{2}\right) d E_{A_{1}} d E_{A_{2}}, \\
p_{j} \in\left(R\left[t_{j}\right]\right)_{+}, \quad j=1,2 .
\end{gathered}
$$

In particular, $f$ is a linear positive operator on the subspace generated by the products of even polynomials in each variable, which is a majorizing subspace in $X_{2}$. This yields the existence of a positive extension $F$ of $f$ to the whole space $X_{2}$. The positivity of $F$ and the uniform convergence of $\left(p_{m}\right)_{m}$ to $x_{2} \geq 0$ on $S\left(A_{1}\right) \times S\left(A_{2}\right)$ lead to

$$
\begin{aligned}
F\left(x_{2}\right) & =\lim F\left(p_{m}\right)=\lim f\left(p_{m}\right) \\
& \leq \lim \int_{S\left(A_{1}\right) \times S\left(A_{2}\right)} p_{m, 1}\left(t_{1}\right) p_{m, 2}\left(t_{2}\right) d E_{A_{1}} d E_{A_{2}} \\
& =\int_{S\left(A_{1}\right) \times S\left(A_{2}\right)} x_{2}\left(t_{1}, t_{2}\right) d E_{A_{1}} d E_{A_{2}} .
\end{aligned}
$$

The last relation (a) follows too. This concludes the proof.

\section{Applying the Solutions of the Abstract Moment Problems to Some Classical Moment Problems}

The results of this section follow the papers in $[7,8]$. In the next theorem, $X$ will be the space of all absolutely convergent power series:

$$
\begin{aligned}
& \varphi(z)=\sum_{j=0}^{\infty} \alpha_{j} z^{j}, \quad|z|<b, \\
& X_{+}=\left\{\varphi \in X ; \alpha_{j} \in R_{+} \quad \forall j \in Z_{+}\right\}, \\
& X=X_{+}-X_{+} .
\end{aligned}
$$

One assumes that the elements of the space $X$ are continuous in the closed disk $\{|z| \leq b\}$. Let $H$ be a Hilbert space, $U_{0}$ a selfadjoint operator acting on $H$, and $Y=Y\left(U_{0}\right)$ the ordercomplete vector lattice and commutative Banach algebra of selfadjoint operators defined in Section 2 (see also $[6,14]$ ). We denote that $\varphi_{j}(z)=z^{j},|z| \leq b, j \in Z_{+}$.

Theorem 14. Let $X, H, U_{0}, Y, \varphi_{j}$ be as previously mentioned. Assume that $b>1$ and let $A \in Y,\|A\|<b$. Let $\varepsilon>0$ and $\left\{B_{j}\right\}_{j \in Z_{+}} \subset Y$. The following statements are equivalent:

(a) there exists a linear operator $F$ applying $X$ into $Y$ such that

$$
\begin{aligned}
& F\left(\varphi_{j}\right)=B_{j} \quad \forall j \in Z_{+}, \\
& \varphi(A)-\varepsilon \cdot \varphi(I) \leq F(\varphi) \leq \varphi(A)+\varepsilon \cdot \varphi(I) \quad \forall \varphi \in X_{+}, \\
& \|F(\varphi)\| \leq\|\varphi\|+\varepsilon \cdot|\varphi(1)|, \quad \varphi \in X ;
\end{aligned}
$$

(b) one has

$$
A^{j}-\varepsilon \cdot I \leq B_{j} \leq A^{j}+\varepsilon \cdot I, j \in Z_{+} .
$$

Proof. (a) $\Rightarrow(\mathrm{b})$ is obvious, since from (a) we derive

$$
\begin{aligned}
B_{j} & =F\left(\varphi_{j}\right) \in\left[\varphi_{j}(A)-\varepsilon \cdot \varphi_{j}(I), \varphi_{j}(A)+\varepsilon \cdot \varphi_{j}(I)\right] \\
& =\left[A^{j}-\varepsilon \cdot I, A^{j}+\varepsilon \cdot I\right], \quad j \in Z_{+} .
\end{aligned}
$$

(b) $\Rightarrow$ (a). One applies Theorem 4 to $x_{j}=\varphi_{j} \in X, j \in Z_{+}$. We have

$$
\begin{aligned}
& \sum_{j \in F} \lambda_{j} \varphi_{j}=\psi_{2}-\psi_{1}=\sum_{j \in Z_{+}} a_{j} \varphi_{j}-\sum_{j \in Z_{+}} b_{j} \varphi_{j}, \quad a_{j}, b_{j} \geq 0 \\
& \Longrightarrow-\left(a_{j}+b_{j}\right) \leq-b_{j} \leq \lambda_{j} \leq a_{j} \leq a_{j}+b_{j} \\
& \Longrightarrow\left|\lambda_{j}\right| \leq a_{j}+b_{j}, \quad j \in F \\
& \Longrightarrow \lambda_{j} B_{j} \leq \lambda_{j} A^{j}+\varepsilon \cdot \lambda_{j} I, \quad \forall j \in F^{+}, \\
& \lambda_{j} B_{j} \leq \lambda_{j} A^{j}-\varepsilon \cdot \lambda_{j} I, \quad \forall j \in F^{-}, \\
& F^{+}=\left\{j \in F ; \lambda_{j} \geq 0\right\}, \quad F^{-}=\left\{j \in F ; \quad \lambda_{j}<0\right\} .
\end{aligned}
$$


The preceding relations yield

$$
\begin{aligned}
\sum_{j \in F} \lambda_{j} B_{j} \leq & \sum_{j \in F^{+}} \lambda_{j} A^{j}+\varepsilon \cdot\left(\sum_{j \in F^{+}} \lambda_{j}\right) \cdot I \\
& +\sum_{j \in F^{-}} \lambda_{j} A^{j}-\varepsilon \cdot\left(\sum_{j \in F^{-}} \lambda_{j}\right) \cdot I \\
= & \sum_{j \in F} \lambda_{j} A^{j}+\varepsilon \cdot\left(\sum_{j \in F}\left|\lambda_{j}\right|\right) \cdot I \\
= & \psi_{2}(A)-\psi_{1}(A)+\varepsilon \cdot\left(\sum_{j \in F}\left|\lambda_{j}\right|\right) \cdot I \\
\leq & \psi_{2}(A)-\psi_{1}(A)+\varepsilon \cdot\left(\sum_{j \in Z_{+}}\left|\lambda_{j}\right|\right) \cdot I \\
= & F_{2}\left(\psi_{2}\right)-F_{1}\left(\psi_{1}\right) \cdot \psi_{2}(A)-\psi_{1}(A)+\varepsilon \cdot\left(\sum_{j \in Z_{+}}\left(a_{j}+b_{j}\right)\right) \cdot I \\
= & \psi_{2}(A)-\psi_{1}(A)+\varepsilon \cdot \sum_{j \in Z_{+}}\left(a_{j}+b_{j}\right) \cdot I \\
& \left.-\sum_{j \in Z_{+}} a_{j}\right) \cdot I
\end{aligned}
$$

Theorem 4 implies the existence of a linear operator

$$
\begin{aligned}
& F \in L(X, Y), \quad F_{1} \leq F \leq F_{2} \text { on } X_{+}, \\
& F\left(\varphi_{j}\right)=B_{j} \quad \forall j \in Z_{+} .
\end{aligned}
$$

One obtains

$$
\begin{aligned}
|F(\varphi)| & \leq\left|F\left(\varphi_{+}\right)\right|+\left|F\left(\varphi_{-}\right)\right| \\
& \leq \varphi_{+}(A)+\varepsilon \cdot \varphi_{+}(I)+\varphi_{-}(A)+\varepsilon \cdot \varphi_{-}(I) \\
& =|\varphi(A)|+\varepsilon|\varphi(I)| \leq\|\varphi\| \cdot I+\varepsilon \cdot|\varphi(1)| \cdot I \\
& \Longrightarrow\|F(\varphi)\| \leq\|\varphi\|+\varepsilon \cdot|\varphi(1)|, \quad \forall \varphi \in X .
\end{aligned}
$$

This concludes the proof.

In the next theorems, the notion of a resolvent appears naturally during the proofs.

Theorem 15. Let $b>1, X, Y,\left\{\varphi_{j}\right\}_{j}$ be as previously mentioned. Let $A \in Y, S(A) \subset(0, b),\left\{B_{j}\right\}_{j \in Z_{+}} \subset Y, \varepsilon>0$. Assume that

$$
0 \leq B_{j} \leq A^{j}+\varepsilon \cdot I \quad \forall j \in Z_{+} .
$$

Then, there exists a linear positive operator $F \in L_{+}(X, Y)$, such that

$$
\begin{aligned}
F\left(\varphi_{j}\right) & =B_{j} \quad \forall j \in Z_{+}, \\
|F(\varphi)| & \leq\|\varphi\| \cdot\left[\left(I-b^{-1} A\right)^{-1}+\varepsilon \frac{b}{b-1} I\right] \\
& \Longrightarrow\|F\| \leq \frac{b}{b-\|A\|}+\varepsilon \frac{b}{b-1} .
\end{aligned}
$$

Proof. The following implications hold

$$
\begin{aligned}
\sum_{j \in F} \lambda_{j} \varphi_{j} & \leq \varphi=\sum_{j \in Z_{+}} a_{j} \varphi_{j} \Longrightarrow \lambda_{j} \\
& \leq a_{j} \leq\left|a_{j}\right| \leq \frac{\|\varphi\|_{\infty}}{b^{j}}, \quad j \in F \\
& \Longrightarrow \sum_{j \in F} \lambda_{j} B_{j} \leq \sum_{j \in F_{+}} \lambda_{j}\left(A^{j}+\varepsilon \cdot I\right) \\
& \leq\|\varphi\| \cdot\left[\sum_{j \in Z_{+}} b^{-j} A^{j}+\varepsilon \cdot\left(\sum_{j \in Z_{+}} b^{-j}\right) \cdot I\right] \\
& =\|\varphi\| \cdot\left[\left(I-b^{-1} A\right)^{-1}+\varepsilon \cdot \frac{b}{b-1} I\right] \\
& =p(\varphi), \quad \varphi \in X .
\end{aligned}
$$

Note that in the preceding relations the Cauchy inequalities for the function $\varphi$ were used. Application of Theorem 3 leads to the existence of a linear positive operator $F$ from $X$ into $Y$, satisfying the moment conditions, such that $F \leq p$ on $X$. Now, the conclusion follows easily, by using the monotony of the norm on $Y$ :

$$
\begin{aligned}
\|F\| & \leq\left\|\left(I-b^{-1} A\right)^{-1}\right\|+\varepsilon \cdot \frac{b}{b-1} \\
& \leq 1+\frac{\|A\|}{b}+\frac{\|A\|^{2}}{b^{2}}+\cdots+\varepsilon \cdot \frac{b}{b-1} \\
& =\frac{b}{b-\|A\|}+\varepsilon \cdot \frac{b}{b-1} .
\end{aligned}
$$

The proof is complete.

Similar results are valid for the multidimensional moment problem. Let $X_{\bar{\rho}}$ be the space of analytic functions $\varphi$ in the polydisc $T_{\bar{\rho}}=\prod_{k=1}^{n}\left\{\left|z_{k}\right| \leq \rho_{k}\right\}, \bar{\rho}=\left(\rho_{1}, \ldots, \rho_{n}\right)$. Whence,

$$
\begin{aligned}
& \varphi\left(z_{1}, \ldots, z_{n}\right)=\sum_{j \in Z_{+}^{n}} c_{j} z^{j} \\
&=\sum_{j \in Z_{+}^{n}} c_{j} z_{1}^{j_{1}} \cdots z_{n}^{j_{n}}, \quad j=\left(j_{1}, \ldots, j_{n}\right), \\
&\left|z_{j}\right| \leq \rho_{j}, j=1, \ldots, n, \\
& \varphi_{j}\left(z_{1}, \ldots, z_{n}\right)=z_{1}^{j_{1}} \cdots z_{n}^{j_{n}} .
\end{aligned}
$$


The order relation on $X$ is defined by the positive cone

$$
X_{+}=\left\{\varphi \in X ; \operatorname{Rec} c_{j} \geq 0, \operatorname{Im} c_{j} \geq 0\right\} .
$$

Consider an arbitrary real or complex Hilbert space $H, A_{1}, \ldots, A_{n} \in A(H)$ commuting selfadjoint operators $\left(A_{i} A_{j}=A_{j} A_{i}, i, j=1, \ldots, n\right)$. We introduce the usual spaces:

$$
\begin{aligned}
& Y_{1}=\left\{U \in A(H) ; U A_{j}=A_{j} U, j=1, \ldots, n\right\}, \\
& Y=\left\{U \in Y_{1} ; U V=V U, \forall V \in Y_{1}\right\} \\
& Y_{+}=\{U \in Y ;\langle U(h), h\rangle \geq 0, \forall h \in H\} .
\end{aligned}
$$

Theorem 16 (see [8, Theorem 3.1]). With the previous notations, assume that

$$
\rho_{j}>\left\|A_{j}\right\|, \quad j=1, \ldots, n,
$$

and let $\left\{B_{j} ; j \in Z_{+}^{n}\right\} \subset Y$. Consider the following statements:

(a) there exists an $R$-linear positive continuous operator $F$ : $X_{\bar{\rho}} \rightarrow Y$ such that

$$
\begin{aligned}
& F\left(x_{j}\right)=B_{j}, j \in Z_{+}^{n}, \\
& F(\varphi) \leq\|\varphi\|_{\infty} \prod_{k=1}^{n} \rho_{k}\left(\rho_{k} I-A_{k}\right)^{-1}, \\
& \|F\| \leq \prod_{k=1}^{n} \frac{\rho_{k}}{\rho_{k}-\left\|A_{k}\right\|}
\end{aligned}
$$

(b) one has

$$
0 \leq B_{j} \leq A_{1}^{j_{1}} \cdots A_{n}^{j_{n}}, \quad \forall j \in Z_{+}^{n}
$$

(c) one has

$$
\begin{aligned}
& 0 \leq B_{j} \leq \prod_{k=1}^{n} \rho_{k}^{j_{k}+1}\left(\rho_{k} I-A_{k}\right)^{-1}, \\
& \left\|B_{j}\right\| \leq \prod_{k=1}^{n} \frac{\rho_{k}^{j_{k}+1}}{\rho_{k}-\left\|A_{k}\right\|} \quad \forall j=\left(j_{1}, \ldots, j_{n}\right) \in Z_{+}^{n} .
\end{aligned}
$$

Then, $(b) \Rightarrow(a) \Rightarrow(c)$.

Proof. (b) $\Rightarrow$ (a). Let $F \subset Z_{+}^{n}$ be a finite subset and let $\left\{\lambda_{j}\right\}_{j \in F} \subset$ $R$ be such that

$$
\sum_{j \in F} \lambda_{j} \varphi_{j} \leq \varphi=\sum_{j \in Z_{+}^{n}} c_{j} \varphi_{j}
$$

Then, the order relation on $X$ and Cauchy's inequalities yield

$$
\lambda_{j} \leq \operatorname{Rec}_{j} \leq\left|c_{j}\right| \leq \frac{\|\varphi\|_{\infty}}{\rho_{1}^{j_{1}} \cdots \rho_{n}^{j_{n}}}, \quad j=\left(j_{1}, \ldots, j_{n}\right) .
$$

Using the hypothesis (b) and the previous relations, one obtains

$$
\begin{aligned}
\sum_{j \in F} \lambda_{j} B_{j} & \leq \sum_{j \in F^{+}} \lambda_{j} B_{j} \leq \sum_{j \in F^{+}} \lambda_{j} A_{1}^{j_{1}} \cdots A_{n}^{j_{n}} \\
& \leq\|\varphi\|_{\infty}\left(\sum_{j \in Z_{+}^{n}}\left(\frac{A_{1}}{\rho_{1}}\right)^{j_{1}} \cdots\left(\frac{A_{n}}{\rho_{n}}\right)^{j_{n}}\right) \\
& =\|\varphi\|_{\infty}\left(\sum_{j_{1} \in Z_{+}}\left(\frac{A_{1}}{\rho_{1}}\right)^{j_{1}}\right) \cdots\left(\sum_{j_{n} \in Z_{+}}\left(\frac{A_{n}}{\rho_{n}}\right)^{j_{n}}\right) \\
& =\|\varphi\|_{\infty}\left(I-\frac{A_{1}}{\rho_{1}}\right)^{-1} \cdots\left(I-\frac{A_{n}}{\rho_{n}}\right)^{-1} \\
& =\|\varphi\|_{\infty} \prod_{k=1}^{n} \rho_{k}\left(\rho_{k} I-A_{k}\right)^{-1}=p(\varphi), \quad \varphi \in X .
\end{aligned}
$$

Application of Theorem 3 leads to the existence of an $R$-linear positive operator $F$ applying $X$ into $Y$ such that

$$
\begin{aligned}
& F\left(\varphi_{j}\right)=B_{j}, \quad j \in Z_{+}^{n}, \\
& F(\varphi) \leq p(\varphi)=p(-\varphi) \quad \forall \varphi \in X \\
& \quad \Longrightarrow|F(\varphi)| \leq p(\varphi), \quad \varphi \in X .
\end{aligned}
$$

Using the monotony of the norm on $Y$, we deduce

$$
\begin{aligned}
\|F(\varphi)\| \leq & \|\varphi\|_{\infty} \prod_{k=1}^{\infty}\left\|\left(I-\frac{A_{k}}{\rho_{k}}\right)^{-1}\right\| \\
\leq & \|\varphi\|_{\infty} \\
& \times\left[\sum_{j_{1} \in Z_{+}}\left(\frac{\left\|A_{1}\right\|}{\rho_{1}}\right)^{j_{1}}\right] \ldots\left[\sum_{j_{n} \in Z_{+}}\left(\frac{\left\|A_{n}\right\|}{\rho_{n}}\right)^{j_{n}}\right] \\
= & \|\varphi\|_{\infty} \prod_{k=1}^{\infty} \frac{\rho_{k}}{\rho_{k}-\left\|A_{k}\right\|}, \quad \forall \varphi \in X .
\end{aligned}
$$

Thus, the implication (b) $\Rightarrow(\mathrm{a})$ is proved.

$(a) \Rightarrow(c)$ The hypothesis on positivity of the solution $F$ and of functions $\varphi_{j}$ yield

$$
\begin{aligned}
B_{j} & =F\left(\varphi_{j}\right) \leq\left\|\varphi_{j}\right\|_{\infty} \prod_{k=1}^{n} \rho_{k}\left(\rho_{k} I-A_{k}\right)^{-1} \\
& =\prod_{k=1}^{n} \rho_{k}^{j_{k}+1}\left(\rho_{k} I-A_{k}\right)^{-1} \Longrightarrow\left\|B_{j}\right\| \\
& =\left\|F\left(\varphi_{j}\right)\right\| \leq\left\|\varphi_{j}\right\| \prod_{k=1}^{n} \frac{\rho_{k}}{\rho_{k}-\left\|A_{k}\right\|} \\
& =\prod_{k=1}^{n} \frac{\rho_{k}^{j_{k}+1}}{\rho_{k}-\left\|A_{k}\right\|}, \quad j=\left(j_{1}, \ldots, j_{n}\right) \in Z_{+}^{n} .
\end{aligned}
$$

The proof is complete. 
Applying Theorem 16 to the real Hilbert space $H=$ $L_{d t}^{2}([0, \pi / 2]), n=2, A_{k}, k=1,2$ being the multiplication operator with the functions $h_{1}(t)=\sin t, h_{2}(t)=\cos t$, one obtains the following.

Corollary 17. Let $\left\{B_{j} ; j \in Z_{+}^{2}\right\} \subset Y=Y\left(A_{1}, A_{2}\right), \rho>1$, $\rho_{1}=\rho_{2}=\rho$.

Consider the following statements:

(a) there exists $F \in L_{+}(X, Y)$ such that

$$
\begin{array}{r}
F\left(\varphi_{j}\right)=B_{j}, \quad j \in Z_{+}^{2}, \int_{0}^{\pi / 2}(F(\varphi)(h))(t) \cdot h(t) d t \\
\leq\|\varphi\| \rho^{2} \int_{0}^{\pi / 2} \frac{h^{2}(t)}{(\rho-\sin t)(\rho-\cos t)} d t \\
\forall h \in L_{d t}^{2}\left(\left[0, \frac{\pi}{2}\right]\right) ;
\end{array}
$$

(b) one has

$$
\begin{aligned}
0 \leq & \int_{0}^{\pi / 2} B_{j}(h)(t) \cdot h(t) d t \\
\leq & \int_{0}^{\pi / 2} \sin ^{j_{1}} t \cdot \cos ^{j_{2}} t \cdot h^{2}(t) d t \\
& \forall h \in H, \quad j=\left(j_{1}, j_{2}\right) \in Z_{+}^{2} ;
\end{aligned}
$$

(c) one has

$$
\begin{aligned}
0 & \leq \int_{0}^{\pi / 2} B_{\left(j_{1}, j_{2}\right)}(h)(t) \cdot h(t) d t \\
& \leq \rho^{j_{1}+j_{2}+2} \int_{0}^{\pi / 2} \frac{h^{2}(t)}{(\rho-\sin t)(\rho-\cos t)} d t, \quad \forall h \in H .
\end{aligned}
$$

Then, $(b) \Rightarrow(a) \Rightarrow(c)$.

For some related results see [8].

Our next goal is to consider some Markov moment problems in terms of nonnegative sequences with respect to an interval. We recall this notion, that is well known.

Definition 18. A sequence $\left(u_{n}\right)_{n=0}^{\infty}$ in an ordered vector space $Y$ is said to be nonnegative with respect to the interval $I \subset R$ if for any $n \in Z_{+}$, we have

$$
\begin{aligned}
\lambda_{0}+ & \lambda_{1} t+\cdots+\lambda_{n} t^{n} \geq 0 \quad \forall t \in I \\
& \Longrightarrow \lambda_{0} u_{0}+\lambda_{1} u_{1}+\cdots+\lambda_{n} u_{n} \geq 0 \text { in } Y .
\end{aligned}
$$

It is clear that for compact intervals and for $Y=R$, this condition is necessary and sufficient for the existence of a unique positive solution of the moment problem associated to the moments $y_{j}=u_{j} \in R, j \in Z_{+}$. The continuity with respect to the supnorm is also obvious. But no information concerning dominating $L^{1}$ norm for the solution holds. Such information would be useful for integral representation of the extension of the solution to the space $L^{1}(I)$ (see the next results).
Theorem 19. Let $b \in(0, \infty)$. Consider the following statements:

(a) there exists a unique $h \in L^{\infty}([0, b])$ such that

$$
0 \leq h(t) \leq 1 \text { a.e., } \quad \int_{0}^{b} t^{j} h(t) d t=y_{j}, \quad \forall j \in Z_{+} ;
$$

(b) the sequence

$$
\left(1, y_{0}, 2 y_{1}, \ldots, n y_{n-1}, \ldots\right)
$$

is nonnegative with respect to $[0, b]$.

Then, $(b) \Rightarrow(a)$.

Proof. We have

$$
\sum_{j=0}^{n} \lambda_{j} \tau^{j}=\varphi_{2}(\tau)-\varphi_{1}(\tau), \quad \varphi_{1}, \varphi_{2} \in X_{+}, X=L^{1}([0, b])
$$

$$
\begin{aligned}
& \Longrightarrow \lambda_{0} t+\lambda_{1} \frac{t^{2}}{2}+\cdots+\lambda_{n} \frac{t^{n+1}}{n+1} \leq \int_{0}^{b} \varphi_{2}(\tau) d \tau \\
& =F_{2}\left(\varphi_{2}\right)-F_{1}\left(\varphi_{1}\right), \quad\left(F_{1} \equiv 0\right), \quad \forall t \in[0, b] \\
& \Longrightarrow \int_{0}^{b} \varphi_{2} d \tau-\lambda_{0} t-\lambda_{1} \frac{t^{2}}{2}-\cdots-\lambda_{n} \frac{t^{n+1}}{n+1} \geq 0,
\end{aligned}
$$

$\forall t \in[0, b]$

$$
\begin{gathered}
\Longrightarrow \int_{0}^{b} \varphi_{2}(\tau) d \tau-\lambda_{0} y_{0}-\lambda_{1} \frac{2 y_{1}}{2}-\cdots \\
-\lambda_{n} \frac{(n+1) y_{n}}{n+1} \geq 0 \\
\Longrightarrow \sum_{j=0}^{n} \lambda_{j} y_{j} \leq F_{2}\left(\varphi_{2}\right)-F_{1}\left(\varphi_{1}\right) .
\end{gathered}
$$

Application of Theorem $4,(b) \Rightarrow(a)$, leads to the existence of a linear functional $F$ on $X=L^{1}([0, b])$ such that

$$
\begin{aligned}
& F\left(x_{j}\right)=y_{j}, \quad j \in Z_{+}, \\
& 0=F_{1}(\varphi) \leq F(\varphi) \leq F_{2}(\varphi)=\int_{0}^{b} \varphi d t, \quad \forall \varphi \in X_{+} .
\end{aligned}
$$

Using the representation of a linear positive functional on $L^{1}$, there exists

$$
\begin{aligned}
& h \in L^{\infty}([0, b]), 0 \leq h, \\
& F(\varphi)=\int_{0}^{b} \varphi \cdot h d t, \quad \forall \varphi \in L^{1}([0, b]) .
\end{aligned}
$$

From the last equality written for $\varphi=\chi_{B}, B \subset[0, b]$ being a Borel subset, we infer (via measure theory) that

$$
h \leq 1 \text { a.e., } \quad \int_{0}^{b} t^{j} h(t) d t=F\left(x_{j}\right)=y_{j}, \quad \forall j \in Z_{+} .
$$

The proof is complete. 
Lemma 20. Let $h:[a, b] \rightarrow R$ be a positive function in $L^{1}([a$, $b]$ ) and $p:[a, b] \rightarrow R$ a continuous convex function on $[a, b]$. Then, one has

$$
\int_{a}^{b} p(t) h(t) d t \geq\left(\int_{a}^{b} h(t) d t\right) \cdot p\left(\frac{\int_{a}^{b} t h(t) d t}{\int_{a}^{b} h(t) d t}\right) .
$$

Corollary 21. Let $\left(y_{j}\right)_{j \in Z_{+}}$be a sequence of real numbers such that the sequence

$$
\left(1, y_{0}, 2 y_{1}, \ldots, n y_{n-1},(n+1) y_{n}, \ldots\right)
$$

is nonnegative with respect to $[0, b]$ and $y_{0} \neq 0$. Then,

$$
y_{0}^{1-j} y_{1}^{j} \leq y_{j} \leq \frac{b^{j+1}}{j+1}, \quad j \in Z_{+}, \quad y_{1} \leq b \cdot y_{0} .
$$

Proof. Applying Theorem 19 and Lemma 20 to $p(t)=t^{j}, t \in$ $[0, b], j \in Z_{+}$, one obtains

$$
\begin{aligned}
& y_{j}=\int_{0}^{b} t^{j} h(t) d t \leq \int_{0}^{b} t^{j} d t=\frac{b^{j+1}}{j+1}, \\
& y_{j} \geq \frac{\left(\int_{0}^{b} t h(t) d t\right)^{j}}{\left(\int_{0}^{b} h(t) d t\right)^{j-1}}=y_{0}^{1-j} \cdot y_{1}^{j}, \quad j \in Z_{+}, \\
& y_{1}=\int_{0}^{b} t \cdot h(t) d t \leq b \int_{0}^{b} h(t) d t=b \cdot y_{0} .
\end{aligned}
$$

The proof is finished.

\section{Extreme Points and the Markov Moment Problem}

The aim of this section is to apply some ideas from [20, 26,27 ] in solving truncated moment problems and their connection to the full moment problem. The results of this section are new. The following theorem concerns the finite Markov moment problem considered in [20], also using some results from [27]. Let us denote that

$$
\psi_{j}(t)=j \cdot t^{j-1}, \quad t \in[0, b], j \in \mathbf{N}
$$

Theorem 22. For a given family of numbers $\left(m_{j}\right)_{j=1}^{n}$, consider the following statements: (a), (b), and (c):

(a) there exists $h \in L^{\infty}([0, b])$ such that

$$
0 \leq h(t) \leq 1 \text { a.e., } \quad m_{j}=j \int_{0}^{b} t^{j-1} h(t) d t, \quad j=1,2, \ldots, n ;
$$

(b) for any family of scalars $\left(\lambda_{j}\right)_{j=0}^{n}$, one has:

$$
\sum_{j=1}^{n} \lambda_{j} m_{j} \leq \sum_{j=1}^{n} \lambda_{j} b^{j}
$$

(c) there exists a Borel subset E such that

$$
\int_{B} j \cdot t^{j-1} d t=m_{j}, \quad j=1, \ldots, n .
$$

Then, $(b) \Rightarrow(a) \Leftrightarrow(c)$

Proof. Let the point (b) be accomplished and assume that

$$
\sum_{j=1}^{n} \lambda_{j} \cdot j \cdot t^{j-1}=\varphi_{2}-\varphi_{1}, \quad \varphi_{j} \in\left(L^{1}([0, b])\right)_{+} .
$$

Then the integration on $[0, b]$ yields

$$
\sum_{j=1}^{n} \lambda_{j} b^{j} \leq \int_{0}^{b} \varphi_{2}(t) d t=F_{2}\left(\varphi_{2}\right) \Longrightarrow \sum_{j=1}^{n} \lambda_{j} m_{j} \leq \int_{0}^{b} \varphi_{2}(t) d t .
$$

Application of Theorem 4 to $F_{1} \equiv 0, F_{2}$ defined previously, leads to the existence of a linear positive form $F$ on $L^{1}([0, b])$ such that

$$
\begin{aligned}
F\left(\psi_{j}\right) & =m_{j}, \quad j=1, \ldots, n, F(\psi) \\
& \leq \int_{0}^{b} \psi \cdot d t, \quad \psi \in\left(L^{1}([0, b])\right)_{+} \\
& \Longrightarrow|F(\varphi)| \leq F\left(\varphi^{+}\right)+F\left(\varphi^{-}\right) \\
& \leq \int_{0}^{b}|\varphi(t)| \cdot d t, \quad \varphi \in L^{1}([0, b]) .
\end{aligned}
$$

If characteristic functions of Borel subsets stand for $\psi$, the conclusion (a) follows by measure theory [28]. The implication $(\mathrm{a}) \Rightarrow(\mathrm{c})$ is a consequence of equality (15.14) in [27] (see also [27, Exercise 2.57]). The set of values for the control function $u$, namely, $[-1,1]$, is replaced by $[0,1]$, the set of values for $h$, that stands for the control function $u$. The extreme points of the positive part of the unit ball of $L^{\infty}([0, b])$ are the characteristic functions of measurable sets. The converse is obvious.

Corollary 23. Under the equivalent conditions (a), (c) of Theorem 22, there are sequences

$$
y_{1, n}<x_{1, n}<y_{2, n}<x_{2, n}<\cdots<y_{l, n}<x_{l, n}<\cdots, \quad n \in \mathbf{N}
$$

such that the following relations hold:

$$
m_{k}=\inf _{n \in \mathbf{N}}\left(\sum_{j=1}^{\infty}\left(x_{j, n}^{k}-y_{j, n}^{k}\right)\right), \quad k=1, \ldots, n .
$$

Proof. One uses the fact that any Borel subset is a joint of a $G_{\delta}$ set and a set of measure zero [28].

Remark 24. In order to approximate the numbers $x_{j, n}^{k}, y_{j, n}^{k}$, one can make use of Fourier approximate expansion of $h$ with respect to the orthonormal sequence attached to the 
functions $k t^{k-1}$ via Gram-Schmidt algorithm, also using the values of the moments $m_{k}$. Thus, one obtains a smooth approximation $\tilde{h}$ of $h$, and the intervals of ends $y_{l, n}, x_{l, n}$ are connected components of the open sets of the form

$$
\left\{\frac{j_{l}}{2^{p(n)}}<\tilde{h}(t)<\frac{j_{l}+1}{2^{p(n)}}\right\} .
$$

Remark 25. A similar result to that of Theorem 22 in several dimensions holds, with the same proof. We state it for the two-dimensional case.

Theorem 26. Let $\left(m_{\left(j_{1}, j_{2}\right)}\right)_{\substack{1 \leq j_{1} \leq n_{1} \\ 1 \leq j_{2} \leq n_{2}}}$ be a given family of real numbers and consider the functions

$$
\begin{aligned}
& \psi_{\left(j_{1}, j_{2}\right)}\left(t_{1}, t_{2}\right)=j_{1} j_{2} t_{1}^{j_{1}-1} t_{2}^{j_{2}-1}, \quad 1 \leq j_{p} \leq n_{p}, \\
& p=1,2, \quad\left(t_{1}, t_{2}\right) \in K_{2}=\left[0, b_{1}\right] \times\left[0, b_{2}\right] .
\end{aligned}
$$

Consider the following assertions: (a), (b), (c). Then $(b) \Rightarrow(a) \Leftrightarrow(c)$

(a) there exists a Borel function $h, 0 \leq h\left(t_{1}, t_{2}\right) \leq 1$ a.e. such that

$$
\begin{aligned}
& \iint_{K_{2}}\left(\psi_{\left(j_{1}, j_{2}\right)} \cdot h\right)\left(t_{1}, t_{2}\right) d t_{1} d t_{2} \\
& \quad=m_{\left(j_{1}, j_{2}\right)}, \quad 1 \leq j_{1} \leq n_{1}, 1 \leq j_{2} \leq n_{2} ;
\end{aligned}
$$

(b) for any family of scalars $\left\{\lambda_{\left(j_{1}, j_{2}\right)}\right\}_{\substack{1 \leq j_{1} \leq n_{1} \\ 1 \leq j_{2} \leq n_{2}}}$, one has

$$
\sum_{\substack{1 \leq j_{1} \leq n_{1} \\ 1 \leq j_{2} \leq n_{2}}} \lambda_{\left(j_{1}, j_{2}\right)} m_{\left(j_{1}, j_{2}\right)} \leq \sum_{\substack{1 \leq j_{1} \leq n_{1} \\ 1 \leq j_{2} \leq n_{2}}} \lambda_{\left(j_{1}, j_{2}\right)} b_{1}^{j_{1}} b_{2}^{j_{2}}
$$

(c) there exists a Borel subset $B_{2} \subset K_{2}$ such that

$$
\iint_{B_{2}} \psi_{\left(j_{1}, j_{2}\right)}\left(t_{1}, t_{2}\right) d t_{1} d t_{2}=m_{\left(j_{1}, j_{2}\right)}, \quad 1 \leq j_{p} \leq n_{p}, p=1,2 .
$$

Corollary 27. If one of the conditions (a), (c) from Theorem 26 is accomplished, there are sequences

$$
\begin{aligned}
& y_{1, n}<x_{1, n}<\cdots<y_{l, n}<x_{l, n}<\cdots, \\
& v_{1, n}<u_{1, n}<\cdots<v_{l, n}<u_{l, n}<\cdots, \quad n \in \mathbf{N},
\end{aligned}
$$

such that

$$
m_{\left(k_{1}, k_{2}\right)}=\inf _{n \in \mathbf{N}}\left\{\sum_{l \in N}\left(x_{l, n}^{k_{1}}-y_{l, n}^{k_{1}}\right) \cdot\left(u_{l, n}^{k_{2}}-v_{l, n}^{k_{2}}\right)\right\} .
$$

Proof. The Borel subset $B_{2}$ is the joint of a $G_{\delta}$ set and a null set. For an open subset $D_{n} \supset B_{2}$, we consider its decomposition into cells [28], used in the construction of Lebesgue measure. If we determine smooth approximations $\widetilde{h}$ of $h$ by means of Gram-Schmidt algorithm for the functions $\psi_{\left(j_{1}, j_{2}\right)}$ and the given moments $m_{\left(j_{1}, j_{2}\right)}$ via Fourier expansion, then the sequences from the present corollary can be determined by means of cell decomposition of the open subsets

$$
\left\{\left(t_{1}, t_{2}\right) ; \frac{m(l)}{2^{p^{(n)}}}<\widetilde{h}\left(t_{1}, t_{2}\right)<\frac{m(l)+1}{2^{p^{(n)}}}\right\} .
$$

Of course, this way one obtains approximations of these numbers.

The previous statements solve the truncated moment problems and sketch an algorithm for determining the numbers $y_{l, n}, x_{l, n}, v_{l, n}, u_{l, n}$. In the last twenty years the truncated moment problem was studied intensively. In [26], the author proves that solving the truncated (not necessarily complex) moment problem for any natural $n$ yields to the solution of the full moment problem. The idea is of passing through the limit, which is allowed by a weakly compact argument. The following theorem proposes a similar construction, by using Krein-Milman theorem. We state it firstly in the onedimensional case, although the several dimensional case one proves similarly.

Theorem 28. With the notations from Theorem 22, let $\left(m_{k}\right)_{k \geq 1}$ be a sequence of real numbers. Consider the following assertions:

(a) there exists a Borel function $h$ such that

$$
0 \leq h(t) \leq 1 \text { a.e., } m_{k}=k \int_{a}^{b} t^{k-1} h(t) d t, \quad k \in \mathbf{N} \backslash\{0\} ;
$$

(b) for any natural number $n \geq 1$, and any $\varepsilon>0$, there exists nonnegative scalars $\beta_{j}, j=1, \ldots, n$, and sequences:

$y_{j, 1}<x_{j, 1}<\cdots<y_{j, l}<x_{j, l}<\cdots, \quad j=1, \ldots, n$

such as

$1-\varepsilon \leq \sum_{j=1}^{n} \beta_{j} \leq 1, \quad m_{k}=\lim _{n}\left(\sum_{j=1}^{n} \beta_{j}\left(\sum_{l}\left(x_{j, l}^{k}-y_{j, l}^{k}\right)\right)\right) ;$

(c) for any $n \in \mathbf{N} \backslash\{0\}$, there exists a Borel subset $B_{n}$ such that

$$
m_{k}=k \int_{B_{n}} t^{k-1} d t, \quad k=1, \ldots, n
$$

(d) for any natural $n \geq 1$ and any $\left\{\lambda_{1}, \ldots, \lambda_{n}\right\} \subset R$, the following relation holds true:

$$
\sum_{k=1}^{n} \lambda_{k} m_{k} \leq \sum_{k=1}^{n} \lambda_{k}\left(b^{k}-a^{k}\right) .
$$

Then $(\mathrm{d}) \Rightarrow(\mathrm{a}) \Leftrightarrow(\mathrm{b}) \Leftrightarrow(\mathrm{c})$. 
Proof. (a) $\Rightarrow(b)$. One applies Krein-Milman Theorem for the weakly compact subset formed by intersecting the unit ball of $L^{\infty}([a, b])$ with the positive cone of the same space. Then, we must have

$$
\begin{gathered}
h=\lim _{n}\left(\sum_{j=1}^{n} \alpha_{j} \chi_{B_{j}}\right)=\lim _{n}\left(\sum_{j=1}^{n} \beta_{j} \chi_{D_{j}}\right), \\
\alpha_{j} \geq 0, \quad \sum_{j=1}^{n} \alpha_{j}=1, \\
\beta_{j}=\frac{m\left(B_{j}\right)}{m\left(D_{j}\right)} \cdot \alpha_{j} \in\left[(1-\varepsilon) \alpha_{j}, \alpha_{j}\right], \\
B_{j} \subset D_{j}, \quad j=1, \ldots, n, D_{j}
\end{gathered}
$$

which are suitably chosen open subsets, where the limit is in the weak topology on $L^{\infty}$, with respect to the dual pair $\left(L^{1}, L^{\infty}\right) ; m$ is the Lebesgue measure. This leads to

$$
m_{k}=k \int_{a}^{b} t^{k-1} h(t) d t=\lim _{n}\left(\sum_{j=1}^{n} \beta_{j} \int_{D_{j}} k t^{k-1} d t\right)
$$

Since each open subset $D_{j}$ has an at most countable decomposition,

$$
D_{j}=\bigcup_{l}\left(y_{j, l}, x_{j, l}\right)
$$

the conclusion (b) follows. For the converse implication, observe that each step function,

$$
\begin{aligned}
& h_{n}:=\sum_{j=1}^{n} \beta_{j} \chi_{D_{j}}, \quad D_{j}=\bigcup_{l}\left(y_{j, l}, x_{j, l}\right), j=1, \ldots, n^{\prime} \\
& \beta_{j} \geq 0, \quad 1-\varepsilon \leq \sum_{j=1}^{n} \beta_{j} \leq 1,
\end{aligned}
$$

is an element of the positive part of the unit ball in $L^{\infty}$, and this subset is weakly compact. Let $h$ be the $(w)$-limit of a subsequence of the sequence $\left(h_{n}\right)_{n}$. Then, we have

$$
m_{k}=\lim _{n} \int_{a}^{b} h_{m_{n}}(t) k \cdot t^{k-1} d t=k \int_{a}^{b} h(t) \cdot t^{k-1} d t,
$$

by Lebesgue dominated convergence theorem. Hence, $(b) \Rightarrow(a)$ is proved. The implication $(a) \Rightarrow(c)$ follows from (a) $\Rightarrow$ (c) of Theorem 22, since a solution of the full moment problem is a solution of all truncated moment problems. The proof of $(c) \Rightarrow(a)$ is similar to that of $(b) \Rightarrow(a)$. It remains to prove that $(\mathrm{d}) \Rightarrow(\mathrm{a})$. This is a consequence of the implication (b) $\Rightarrow$ (a) of Theorem 4. If $J_{0} \subset \mathbf{N} \backslash\{0\}$ is a finite subset and $\left\{\lambda_{j} ; j \in J_{0}\right\} \subset R$, then the following implications hold true:

$$
\begin{aligned}
& \sum_{j \in J_{0}} \lambda_{j} \cdot j \cdot t^{j-1}=\varphi_{2}-\varphi_{1}, \quad \varphi_{p} \in L_{+}^{1} \\
& \Longrightarrow \sum_{j \in J_{0}} \lambda_{j} \cdot\left(b^{j}-a^{j}\right) \\
& \leq \int_{a}^{b} \varphi_{2} d t=F_{2}\left(\varphi_{2}\right)-F_{1}\left(\varphi_{1}\right), \quad F_{1} \equiv 0 \\
& \Longrightarrow \sum_{j \in J_{0}} \lambda_{j} \cdot m_{j} \leq \int_{a}^{b} \varphi_{2} d t=F_{2}\left(\varphi_{2}\right) .
\end{aligned}
$$

Application of Theorem 4 leads to the existence of a linear functional $F$ on $L^{1}([a, b])$, verifying

$$
\begin{aligned}
& F\left(k t^{k-1}\right)=m_{k}, \quad k \geq 1, k \in \mathbf{N}, \\
& 0 \leq F(\psi) \leq \int_{a}^{b} \psi d t, \quad \forall \psi \in L_{+}^{1}([a, b]) .
\end{aligned}
$$

Now, the conclusion follows by measure theory [28].

Remark 29. For the full moment problem, the following algorithm holds in determining $y_{j, l}, x_{j, l}$.

Step 1. Approximating the function $h$.

Let $\left(e_{n}\right)_{n \geq 1}$ be a Hilbert base constructed by the aid of Gram-Schmidt procedure, applied to the system of linearly independent functions

$$
\varphi_{n}(t)=n \cdot t^{n-1}, \quad n \in \mathbf{N} \backslash\{0\} .
$$

Then, for each fixed $n \geq 1$, one has

$$
e_{n}=\sum_{j=1}^{n} a_{j}^{(n)} \varphi_{j} \Longrightarrow\left\langle h, e_{n}\right\rangle=\sum_{j=1}^{n} a_{j}^{(n)}\left\langle h, \varphi_{j}\right\rangle=\sum_{j=1}^{n} a_{j}^{(n)} m_{j},
$$

where the coefficients $a_{j}^{(n)}$ are known from the Gram-Schmidt procedure. Hence, we can determine each Fourier coefficient of $h$ that is, we can approximate $h$ in $L^{2}$-norm by a sequence of polynomial functions $h_{n}, n \geq 1$. Then, there exists a subsequence [28] $h_{k_{n}} \rightarrow h$ pointwise almost everywhere in $[a, b]$.

Step 2. For each $n \in \mathbf{N} \backslash\{0\}$, the subsets,

$$
\left\{t ; \frac{m_{l}}{2^{p}}<h_{k_{n}}(t)<\frac{m_{l}+1}{2^{p}}\right\}, \quad p, m_{l} \in \mathbf{N},
$$

are open subsets. Their connected components have end points approximations of the unknowns $y_{j, l}, x_{j, l}$. From [26], using a weakly compact standard argument, we can obtain $h$ as the limit of a subsequence of $\left(\chi_{B_{n}}\right)_{n}$, where $B_{n}$ are as at point 
(c) of Theorem 28. Considering a suitable open set $D_{k_{n}} \supset B_{k_{n}}$, from (a) one obtains:

$$
\begin{gathered}
m_{k} \approx \int_{D_{k_{n}}} k \cdot t^{k-1} d t \approx \sum_{l}\left(x_{n, l}^{k}-y_{n, l}^{k}\right), \\
\left.D_{k_{n}}=\bigcup_{l}\right] y_{n, l}, x_{n, l}[.
\end{gathered}
$$

Note that all the results of this section can be adapted to the multidimensional moment problem, with similar proofs.

\section{Distanced Convex Subsets: Extension of Linear Operators and the Moment Problem}

The case when the subset $A$ from Theorem 1 is distanced with respect to the subspace $S$, while the restriction involving $B$ is missing, has a nice geometric meaning and leads to interesting results concerning the extension of linear functionals and operators (see [5]).

If $V$ is a convex neighborhood of the origin in a locally convex space, we denote by $p_{V}$ the gauge attached to $V$.

Theorem 30. Let $X$ be a locally convex space, $Y$ an ordercomplete vector lattice with strong order unit $u_{0}$, and $S \subset X$ a vector subspace. Let $A \subset X$ be a convex subset with the following qualities:

(i) there exists a neighborhood $V$ of the origin such that $(S+V) \cap A=\Phi(A$ and $S$ are distanced $) ;$

(ii) $A$ is bounded.

Then, for any equicontinuous family of linear operators $\left\{f_{j}\right\}_{j \in J} \subset L(S, Y)$ and for any $\tilde{y} \in Y_{+} \backslash\{0\}$, there exists an equicontinuous family $\left\{F_{j}\right\}_{j \in J} \subset L(X, Y)$ such that

$$
\left.F_{j}\right|_{S}=f_{j},\left.\quad F_{j}\right|_{A} \geq \tilde{y}, \quad \forall j \in J .
$$

Moreover, if $V$ is a neighborhood of the origin such that

$$
\begin{aligned}
& f_{j}(V \cap S) \subset\left[-u_{0}, u_{0}\right], \quad(S+V) \cap A=\Phi, \\
& 0<\alpha \in R \text { s.t. }\left.p_{V}\right|_{A} \leq \alpha, \quad \alpha_{1}>0 \text { s.t. } \tilde{y} \leq \alpha_{1} u_{0},
\end{aligned}
$$

then the following relations hold:

$$
F_{j}(x) \leq\left(1+\alpha+\alpha_{1}\right) p_{V}(x) \cdot u_{0}, x \in X, j \in J .
$$

The last relation of Theorem 30 gives a relationship between an upper bound and the lower bound of $F_{j}, j \in J$. The next result is a consequence of the preceding one, in terms of the moment problem. The topology on $Y$ is the norm topology for which the unit closed ball is the interval $\left[-u_{0}, u_{0}\right]$.

\section{Theorem 31. Let}

$$
\begin{aligned}
& 0<b \in R, \quad X=C([0, b]) \\
& x_{j}(t)=t^{j}, \quad j \in Z_{+}, \quad j \geq 1, \quad t \in[0, b] \\
& \left\{\varphi_{k}\right\}_{k \in Z_{+}} \subset X, \quad\left\|\varphi_{k}\right\| \leq 1, \quad \varphi_{0} \equiv 1, \quad \varphi_{k}(0)=1, \quad k \in Z_{+} .
\end{aligned}
$$

Let $Y$ be an order-complete vector lattice with strong order unit $u_{0}$ and $\left(y_{n}\right)_{n \geq 1}$ a sequence in $Y$ such that $\left(u_{0}, y_{1}, y_{2}, \ldots\right)$ is positive with respect to $[0, b]$. Then, for any $\alpha_{1} \in R_{+}$, there exists $F \in L(X, Y)$ such that

$$
\begin{aligned}
& F\left(x_{j}\right)=y_{j}, \quad j \in Z_{+}, j \geq 1, \\
& F\left(\varphi_{k}\right) \geq \alpha_{1} u_{0}, \quad k \in Z_{+}, \\
& F(x) \leq\left(2+\alpha_{1}\right) \cdot\|x\| \cdot u_{0}, \quad x \in X .
\end{aligned}
$$

Moreover, if $\alpha_{1} \geq 1$ and $Y$ is endowed with a linear topology such that its positive cone is closed and normal, then $F$ is continuous and positive.

Proof. Let

$$
S=S p\left\{x_{j} ; j \in Z_{+}, j \geq 1\right\}, \quad A=\operatorname{co}\left\{\varphi_{k} ; k \in Z_{+}\right\} .
$$

For all $p \in S, a \in A$, we have

$$
\|p-a\| \geq|p(0)-a(0)|=1,
$$

so that $d(S, A) \geq 1$ holds. This leads to $(S+B(0,1)) \cap A=\Phi$, so we can take in Theorem $30 V=B(0,1), p_{V}=\|\cdot\|_{\infty}$. From the hypothesis on $\left\|\varphi_{k}\right\|, k \in Z_{+}$, we infer that

$$
\left\|\varphi_{k}\right\| \leq\left. 1 \quad \forall k \in Z_{+} \Longrightarrow\|\cdot\|\right|_{A} \leq 1 \Longrightarrow \alpha:=1,
$$

where $\alpha$ is the constant from Theorem 30. We define that

$$
\tilde{y}:=\alpha_{1} \cdot u_{0}, \quad f\left(\sum_{j=1}^{n} \lambda_{j} x_{j}\right):=\sum_{j=1}^{n} \lambda_{j} y_{j} .
$$

Let $\sum_{j=1}^{n} \lambda_{j} x_{j} \in S \cap B(0,1)$. Using the hypothesis on the sequence which is positive with respect to $[0, b]$, this yields

$$
\begin{aligned}
& \sup \left\{\left|\sum_{j=1}^{n} \lambda_{j} t^{j}\right| ; t \in[0, b]\right\}<1 \\
& \Longrightarrow \sum_{j=1}^{n} \lambda_{j} t^{j}+1>0, \quad 1-\sum_{j=1}^{n} \lambda_{j} t^{j}>0 \\
& \Longrightarrow u_{0}+\sum_{j=1}^{n} \lambda_{j} y_{j} \geq 0, \quad u_{0}-\sum_{j=1}^{n} \lambda_{j} y_{j} \geq 0 \\
& \Longrightarrow f\left(\sum_{j=1}^{n} \lambda_{j} x_{j}\right)=\sum_{j=1}^{n} \lambda_{j} y_{j} \in\left[-u_{0}, u_{0}\right] \\
& \Longrightarrow f(S \cap B(0,1)) \subset\left[-u_{0}, u_{0}\right] .
\end{aligned}
$$

The conclusion is that $f$ verifies the conditions in the statement of Theorem 30. Application of the latter theorem leads to the existence of an extension $F$ of $f$ such that

$$
\left.F\right|_{A} \geq \alpha_{1} y_{0}, \quad F(x) \leq\left(2+\alpha_{1}\right) \cdot\|x\| \cdot u_{0}, \quad x \in X .
$$

Whence, the first part of the theorem is proved. It remains to prove the continuity and positivity of the extension $F$, 
under the hypothesis mentioned in the statement. Replacing $x$ by $-x$, it is easy to see that

$$
|F(x)| \leq\left(2+\alpha_{1}\right) \cdot\|x\| \cdot u_{0}, \quad x \in C([0, b]) .
$$

From these relations, also using that the positive cone $Y_{+}$is normal, the continuity of $F$ follows. In order to prove that $F$ is positive, it is sufficient to show that $F(p) \geq 0$ for all positive polynomials $p$. Hypothesis yields

$$
\begin{aligned}
p(t) & =\lambda_{0}+\sum_{j=1}^{n} \lambda_{j} t^{j} \geq 0 \quad \forall t \in[0, b] \\
& \Longrightarrow \lambda_{0} u_{0}+\sum_{j=1}^{n} \lambda_{j} y_{j} \geq 0 \\
& \Longrightarrow F(p)=\lambda_{0} F\left(\varphi_{0}\right)+\sum_{j=1}^{n} \lambda_{j} y_{j} \\
& \geq \lambda_{0}\left(\alpha_{1} \cdot u_{0}-u_{0}\right)=\lambda_{0}\left(\alpha_{1}-1\right) \cdot u_{0} \geq 0 .
\end{aligned}
$$

The proof is complete.

A consequence of Theorem 31 is the following one.

Corollary 32. Let $H$ be a Hilbert space, $A \in A(H)$ a positive selfadjoint operator, and $Y=Y(A)$ the commutative algebra of selfadjoint operators defined in Section 2. Let $b \in R_{+}$be such that the spectrum $S(A) \subset[0, b]$ and $\alpha_{1} \geq 1$. Then, there exists a nondecreasing mapping $\sigma:[0, b] \rightarrow Y$ such that

$$
\begin{aligned}
& \int_{0}^{b} t^{j} d \sigma(t)=A^{j}, \quad j \in Z_{+}, j \geq 1, \\
& \int_{0}^{b} e^{-k t} d \sigma(t) \geq \alpha_{1} I, \quad k \in Z_{+}, \\
& \int_{0}^{b} x(t) d \sigma(t) \leq\left(2+\alpha_{1}\right) \cdot\|x\| \cdot I, \quad x \in C([0, b]) .
\end{aligned}
$$

In particular, for such a mapping $\sigma$, the following relation holds:

$$
\alpha_{1}+1 \leq\|\sigma(b)-\sigma(0)\|+\|\exp (-k A)\|, \quad k \in Z_{+} .
$$

Proof. We apply Theorem 31 to $\varphi_{k}(t)=e^{-k t}, k \in Z_{+}, t \in$ $[0, b], u_{0}=I$. The sequence $\left(I, A, A^{2}, \ldots, A^{n}, \ldots\right)$ is positive with respect to $[0, b]$ because of the positivity of the spectral measure attached to $A$. Due to the hypothesis of Theorem 31 being accomplished, there exists a positive continuous solution $F$ satisfying the relations in the statement of the latter theorem. This solution applies norm-bounded subsets into order-bounded subsets of $Y(A)$. Application of [14, page 272], yields the existence of a "representing" nondecreasing mapping $\sigma$, such that the vector measure $d \sigma$ represents $F$. Next, we prove the last relation of the statement:

$$
\begin{aligned}
\alpha_{1} I \leq \int_{0}^{b} e^{-k t} d \sigma & =\sigma(b)-\sigma(0)+\sum_{m=1}^{\infty} \frac{(-k)^{m}}{m !} A^{m} \\
& =\sigma(b)-\sigma(0)-I+\exp (-k A) .
\end{aligned}
$$

Further computation yields

$$
\begin{aligned}
\left(\alpha_{1}+1\right) I & \leq \sigma(b)-\sigma(0)+\exp (-k A) \Longrightarrow \alpha_{1}+1 \\
& \leq\|\sigma(b)-\sigma(0)\|+\|\exp (-k A)\|, \quad k \in Z_{+}
\end{aligned}
$$

The proof is complete.

Application of Theorem 31 to positive sequences of real numbers with respect to $[0, b]$, the functions $\varphi_{k}, k \in Z_{+}$being as in Corollary 32, yields the following consequence, which we state without proof.

Corollary 33 (see $\left[5\right.$, Corollary 3.3]). Let $\left(1, y_{1}, y_{2}, \ldots\right)$ be a sequence of real numbers, which is positive with respect to $[0, b]$, $b>0$ being arbitrary. Then, the following inequalities hold:

$$
y_{1} \leq 3^{(j-1) / j} \cdot y_{j}^{1 / j}, \quad j \in Z_{+}, j \geq 1
$$

In particular, one has: $y_{1} \leq 3 \liminf y_{j}^{1 / j}$.

Next, we consider the analogue of Theorem 31, for a space $X$ of analytic functions. Let $b>1$ and $X$ be the space of all functions $\varphi$ which can be represented as an absolutely convergent series,

$$
\varphi(z)=\sum_{j=0}^{\infty} \lambda_{j} z^{j}, \quad|z|<b, \lambda_{j} \in R, \varphi
$$

being continuous in the closed disc $|z| \leq b$. Let $\varphi_{j}(z)=z^{j}$, $j \in Z_{+}, Y=L^{\infty}(\Omega),(\Omega, \mu)$ being a measurable space, and $u_{0}(\omega)=1$, for all $\omega \in \Omega$.

Theorem 34. Let $\left\{\psi_{k}\right\}_{k \in Z_{+}} \subset X$ be a sequence such that

$$
\left\|\psi_{k}\right\|_{\infty} \leq M, \quad \psi_{k}(0)=1, \quad \forall k \in Z_{+} .
$$

Assume that $b>1$ and let $\left(y_{j}\right)_{j \geq 1}$ be a sequence in $Y$ such that

$$
\left\|y_{j}\right\| \leq b-1, \quad j \geq 1, \quad j \in Z .
$$

Then, for any $\tilde{y} \in Y_{+} \backslash\{0\}$, there exists $F \in L(X, Y)$ such that

$$
\begin{aligned}
& F\left(\varphi_{j}\right)=y_{j}, \quad j \geq 1, \quad F\left(\psi_{k}\right) \geq \tilde{y}, \quad k \in Z_{+}, \\
& F(x) \leq\left(1+M+\|\tilde{y}\|_{\infty}\right) \cdot\|x\|_{\infty} \cdot u_{0}, \quad x \in X .
\end{aligned}
$$

Proof. If $S=S p\left\{\varphi_{j} ; j \geq 1\right\}, A=\operatorname{co}\left\{\psi_{k} ; k \in Z_{+}\right\}$, we have

$$
\begin{array}{ll}
d(S, A) \geq 1, & (S+B(0,1)) \cap A=\Phi, \\
V=B(0,1), & p_{V}=\|\cdot\|_{\infty} .
\end{array}
$$

Let $\varphi \in B(0,1) \cap S, \varphi=\sum_{j=1}^{n} \lambda_{j} \varphi_{j},\|\varphi\|<1$. Using the Cauchy inequalities, we derive

$$
\left|\lambda_{j}\right| \leq \frac{\|\varphi\|}{b^{j}}<\frac{1}{b^{j}}, \quad j \in\{1,2, \ldots, n\} .
$$


Define $f\left(\sum_{j=1}^{n} \lambda_{j} \varphi_{j}\right)=\sum_{j=1}^{n} \lambda_{j} y_{j}, n \in Z, n \geq 1, \lambda_{j} \in R$. Using also the hypothesis on $\left\|y_{j}\right\|, j \geq 1$, one obtains

$$
\begin{aligned}
\left|\sum_{j=1}^{n} \lambda_{j} y_{j}\right| & \leq \sum_{j=1}^{n}\left|\lambda_{j}\right| \cdot\left|y_{j}\right| \leq\left(\sum_{j=1}^{n}\left(\frac{1}{b^{j}}\right) \cdot\left\|y_{j}\right\|_{\infty}\right) \cdot u_{0} \\
& \leq\left(\sum_{j=1}^{\infty}\left(\frac{1}{b^{j}}\right)\right) \cdot(b-1) u_{0}=u_{0} .
\end{aligned}
$$

Whence, $\varphi=\sum_{j=1}^{n} \lambda_{j} \varphi_{j} \in S \cap B(0,1) \Rightarrow f(\varphi) \in\left[-u_{0}, u_{0}\right]$. Since

$$
\left.\|\cdot\|\right|_{A} \leq M, \quad \tilde{y} \leq\|\tilde{y}\|_{\infty} \cdot u_{0},
$$

one takes in Theorem $30 \alpha=M, \alpha_{1}=\|\tilde{y}\|_{\infty}$. Now, the conclusion follows via Theorem 30 , for which all requirements of the hypothesis are accomplished.

\section{Geometric Aspects Related to the Moment Problem}

In the first part of this section, we give sufficient conditions for the existence of a solution, in a very general setting. The method works in an arbitrary measurable space, which may not involve polynomials. In the particular case of subsets of $R^{n}$, the form of positive polynomials is not necessarily used.

Theorem 35. Let $(T, v)$ be a measurable space, where $v$ is a positive $\sigma$-finite measure on T. Let $X=L_{v}^{1}(T),\left\{x_{j}\right\}_{j \in J} \subset X$, $\left\{y_{j}\right\}_{j \in J} \subset R$. Consider the following statements:

(a) there exists $h \in L_{v}^{\infty}(T)$ such that

$$
\begin{gathered}
\int_{T} x_{j}(t) \cdot h(t) d v=y_{j}, j \in J, \\
-1 \leq h(t) \leq 1 \quad v \text {-a.e.; }
\end{gathered}
$$

(b) for any finite subset $J_{0} \subset J$ and any $\left\{\lambda_{j} ; j \in J_{0}\right\} \subset R$, one has

$$
\begin{aligned}
& \sum_{i, j \in J_{0}} \lambda_{i} \lambda_{j} y_{i} y_{j} \\
& \quad \leq \sum_{i, j \in J_{0}} \lambda_{i} \lambda_{j} \int_{T} x_{i}(t) d v \cdot \int_{T} x_{j}(t) d v ;
\end{aligned}
$$

(c) for any finite subset $J_{0} \subset J$ and any $\left\{\lambda_{j} ; j \in J_{0}\right\} \subset R$, one has

$$
\begin{aligned}
& \sum_{i, j \in J_{0}} \lambda_{i} \lambda_{j} y_{i} y_{j} \\
& \quad \leq \sum_{i, j \in J_{0}}\left|\lambda_{i}\right| \cdot\left|\lambda_{j}\right| \cdot \int_{T}\left|x_{i}(t)\right| d v \cdot \int_{T}\left|x_{j}(t)\right| d v .
\end{aligned}
$$

Then, $(b) \Rightarrow(a) \Rightarrow(c)$.
Proof. (b) $\Rightarrow$ (a). The following implications hold:

$$
\begin{aligned}
& \sum_{j \in J_{0}} \lambda_{j} x_{j}=\varphi_{2}-\varphi_{1}, \quad \varphi_{k} \in X_{+}, k=1,2 \\
& \Longrightarrow-\int_{T} \varphi_{1} d v-\int_{T} \varphi_{2} d v \leq \int_{T} \varphi_{2} d v-\int_{T} \varphi_{1} d v \\
& =\sum_{j \in J_{0}} \lambda_{j} \int_{T} x_{j} d v \leq \int_{T} \varphi_{2} d v+\int_{T} \varphi_{1} d v \\
& =\int_{T} \varphi_{2} d v-\left(-\int_{T} \varphi_{1} d v\right)=F_{2}\left(\varphi_{2}\right)-F_{1}\left(\varphi_{1}\right) \\
& \Longrightarrow\left|\sum_{j \in J_{0}} \lambda_{j} \int_{T} x_{j} d v\right| \leq F_{2}\left(\varphi_{2}\right)-F_{1}\left(\varphi_{1}\right) .
\end{aligned}
$$

On the other hand, the condition (b) can be rewritten as

$$
\begin{aligned}
\left(\sum_{j \in J_{0}} \lambda_{j} y_{j}\right)^{2} & \leq\left(\sum_{j \in J_{0}} \lambda_{j} \int_{T} x_{j} d v\right)^{2} \\
& \Longleftrightarrow\left|\sum_{j \in J_{0}} \lambda_{j} y_{j}\right| \leq\left|\sum_{j \in J_{0}} \lambda_{j} \int_{T} x_{j} d v\right| .
\end{aligned}
$$

From the preceding relation, we conclude

$$
\begin{aligned}
\sum_{j \in J_{0}} \lambda_{j} y_{j} & \leq\left|\sum_{j \in J_{0}} \lambda_{j} y_{j}\right| \\
& \leq\left|\sum_{j \in J_{0}} \lambda_{j} \int_{T} x_{j}(t) d v\right| \leq F_{2}\left(\varphi_{2}\right)-F_{1}\left(\varphi_{1}\right) .
\end{aligned}
$$

Whence, the conditions from the statement of Theorem 4 are accomplished, so that there exists a linear form $F$ on $X$ such that

$$
\begin{aligned}
-\int_{T} \varphi d v= & F_{1}(\varphi) \leq F(\varphi) \leq F_{2}(\varphi) \\
& =\int_{T} \varphi d v \Longrightarrow|F(\varphi)| \leq \int_{T} \varphi d v \quad \forall \varphi \in X_{+} \\
\Longrightarrow|F(\varphi)| & \leq\left|F\left(\varphi^{+}\right)\right|+\left|F\left(\varphi^{-}\right)\right| \\
& \leq \int_{T}\left(\varphi^{+}+\varphi^{-}\right) d v=\int_{T}|\varphi| d v=\|\varphi\|_{1} \Longrightarrow\|F\| \leq 1 .
\end{aligned}
$$

Following the representation of continuous linear functionals on $L^{1}$ spaces, there exist

$$
\begin{aligned}
& h \in L_{v}^{\infty}(T), \quad F(\varphi)=\int_{T} \varphi(t) h(t) d v \quad \forall \varphi \in L_{v}^{1}(T), \\
& \|h\|_{\infty}=\|F\| \leq 1 \Longrightarrow-1 \leq h(t) \leq 1 \quad v \text {-a.e. }
\end{aligned}
$$


We also infer that

$$
y_{j}=F\left(x_{j}\right)=\int_{T} x_{j}(t) h(t) d v, \quad \forall j \in J .
$$

Thus, the proof of the implication (b) $\Rightarrow(a)$ is finished. The implication $(\mathrm{a}) \Rightarrow(\mathrm{c})$ is almost obvious:

$$
\begin{aligned}
\left|\sum_{j \in J_{0}} \lambda_{j} y_{j}\right| & =\left|\sum_{j \in J_{0}} \lambda_{j} \int_{T} x_{j} \cdot h \cdot d v\right| \\
& \leq \sum_{j \in J_{0}}\left|\lambda_{j}\right| \cdot \int_{T}\left|x_{j}(t)\right| \cdot|h(t)| \cdot d v \\
& \leq \sum_{j \in J_{0}}\left|\lambda_{j}\right| \cdot \int_{T}\left|x_{j}(t)\right| \cdot d v .
\end{aligned}
$$

Taking the squares of the two members, the inequality is preserved. The proof is complete.

\section{Corollary 36. Let}

$$
\begin{aligned}
& T=\left\{\left(t_{1}, t_{2}\right) \in R^{2} ; 0 \leq t_{1}<\infty, 0 \leq t_{2} \leq \exp \left(-t_{1}\right)\right\}, \\
& \left\{y_{\left(j_{1}, j_{2}\right)}\right\}_{\left(j_{1}, j_{2}\right) \in Z_{+}^{2}} \subset R .
\end{aligned}
$$

\section{Consider the following statements:}

(a) there exists a Lebesgue measurable function h on $T$ such that

$$
\begin{array}{r}
\int_{T} t_{1}^{j_{1}} t_{2}^{j_{2}} h\left(t_{1}, t_{2}\right) d t_{1} d t_{2}= \\
y_{\left(j_{1}, j_{2}\right)}, \quad \forall\left(j_{1}, j_{2}\right) \in Z_{+}^{2}, \\
-1 \leq h\left(t_{1}, t_{2}\right) \leq 1 \quad \text { a.e.; }
\end{array}
$$

(b) for any finite subset $J_{0} \subset Z_{+}^{2}$ and any $\left\{\lambda_{\left(j_{1}, j_{2}\right)} ;\left(j_{1}, j_{2}\right) \in\right.$ $\left.J_{0}\right\} \subset R$, one has

$$
\begin{aligned}
& \sum_{\left(i_{1}, i_{2}\right),\left(j_{1}, j_{2}\right) \in J_{0}} \lambda_{\left(i_{1}, i_{2}\right)} \lambda_{\left(j_{1}, j_{2}\right)} y_{\left(i_{1}, i_{2}\right)} y_{\left(j_{1}, j_{2}\right)} \\
& \leq \sum_{\left(i_{1}, i_{2}\right),\left(j_{1}, j_{2}\right) \in J_{0}} \lambda_{\left(i_{1}, i_{2}\right)} \lambda_{\left(j_{1}, j_{2}\right)} \frac{i_{1} !}{\left(i_{2}+1\right)^{i_{1}+2}} \cdot \frac{j_{1} !}{\left(j_{2}+1\right)^{j_{1}+2}} .
\end{aligned}
$$

Then, $(b) \Rightarrow(a)$.

Note that $T$ is a closed unbounded and non-semialgebraic subset.

Details can be found in [8]. In [8, Theorem 2.5], the construction of the solution of a moment problem on the ellipse is given.

\section{References}

[1] N. I. Akhiezer, The Classical Moment Problem and Some Related Questions in Analysis, Oliver and Boyd, London, UK, 1965.
[2] C. Ambrozie and O. Olteanu, "A sandwich theorem, the moment problem, finite-simplicial sets and some inequalities," Revue Roumaine de Mathématique Pures et Appliquées, vol. 49, pp. 189-210, 2004.

[3] G. Choquet, Le ProbLème Des Moments, Séminaire D'Initiation À L’AnaLise, Institut Henri Poincaré, Paris, France, 1962.

[4] M. G. Krein and A. A. Nudelman, Markov Moment Problem and Extremal Problems, American Mathematical Society, Providence, RI, USA, 1977.

[5] L. L. Ninulescu and O. Olteanu, "Extension of linear operators, distanced convex sets and the moment problem," Mathematica, vol. 69, no. 1, pp. 81-88, 2004.

[6] L. L. Ninulescu, "Using the solution of an abstract moment problem to solve some classical complex moment problems," Romanian Journal of Pure and Applied Mathematics, vol. 51, pp. 703-711, 2006.

[7] L. Lemnete-Ninulescu, A. Olteanu, and O. Olteanu, "Applications of the solutions of two abstract moment problems to the classical moment problem," Mathematica, vol. 71, no. 2, pp. 173-182, 2006.

[8] J. M. Mihăilă, O. Olteanu, and C. Udrişte, "Markov-type and operator-valued multidimensional moment problems, with some applications," Romanian Journal of Pure and Applied Mathematics, vol. 52, pp. 405-428, 2007.

[9] J. M. Mihăilă, O. Olteanu, and C. Udrişte, "Markov-type moment problems for arbitrary compact and for some noncompact Borel subsets of $R^{n}$," Romanian Journal of Pure and Applied Mathematics, vol. 52, pp. 655-664, 2007.

[10] A. Olteanu and O. Olteanu, "Some unexpected problems of the moment problem," in Proceedings of the 6th Congress of Romanian Mathematicians, vol. 1, pp. 347-355, Editura Academiei Române, Bucharest, Romania, 2009.

[11] O. Olteanu, "Application de théorèmes de prolongement d'opérateurs linéaires au problème des moments et à une generalisation d'un théorème de Mazur-Orlicz,' Comptes Rendus de l’Académie des Sciences, Série I, vol. 313, pp. 739-742, 1991.

[12] O. Olteanu, "Applications of a general sandwich theorem for operators to the moment problem," Revue Roumaine de Mathématique Pures et Appliquées, vol. 1, no. 2, pp. 513-521, 1996.

[13] O. Olteanu, "New aspects of the classical moment problem," Romanian Journal of Pure and Applied Mathematics, vol. 49, pp. 63-77, 2004.

[14] R. Cristescu, Ordered Vector Spaces and Linear Operators, Editura Academiei, Bucharest, and Abacus Press, Kent, UK, 1976.

[15] H. H. Schaefer, Topological Vector Spaces, Springer, Berlin, Germany, 1971.

[16] O. Olteanu, "Convexité et prolongement d’opérateurs linéaires," Comptes Rendus de l'Académie des Sciences, Série I, vol. 286, pp. 511-514, 1978.

[17] O. Olteanu, “Théorèmes de prolongement d’opérateurs linéaires," Revue Roumaine de Mathématique Pures et Appliquées, vol. 28, no. 10, pp. 953-983, 1983.

[18] C. Berg, J. P. R. Christensen, and C. U. Jensen, Harmonic Analysis on Semigroups. Theory of Positive Definite and Related Functions, Springer, Berlin, Germany, 1984.

[19] B. Fuglede, "The multidimensional moment problem," Expositiones Mathematicae, vol. 1, pp. 47-65, 1983.

[20] L. Gosse and O. Runborg, "Existence, uniqueness, and a constructive solution algorithm for a class of finite Markov 
moment problems," SIAM Journal on Applied Mathematics, vol. 68, no. 6, pp. 1618-1640, 2008.

[21] C. Kleiber and J. Stoyanov, "Multivariate distributions and the moment problem," Journal of Multivariate Analysis, vol. 113, pp. 7-18, 2013.

[22] J. Stoyanov, "Stieltjes classes for moment-indeterminate probability distributions," Journal of Applied Probability A, vol. 41, pp. 281-294, 2004.

[23] C. Berg and A. J. Durán, "The fixed point for a transformation of Hausdorff moment sequences and iteration of a rational function," Mathematica Scandinavica, vol. 103, no. 1, pp. 11-39, 2008.

[24] L. Gosse and O. Runborg, "Resolution of the finite Markov moment problem," Comptes Rendus Mathematique, vol. 341, no. 12, pp. 775-780, 2005.

[25] M. Putinar and F. H. Vasilescu, "Problème des moments sur les compacts semi-algébriques," Comptes Rendus de l'Académie des Sciences, Série I, vol. 323, pp. 787-791, 1996.

[26] J. Stochel, "Solving the truncated moment problem solves the full moment problem," Glasgow Mathematical Journal, vol. 43, no. 3, pp. 335-341, 2001.

[27] R. B. Holmes, Geometric Functional Analysis, Springer, Berlin, Germany, 1975.

[28] W. Rudin, Real and Complex Analysis, McGraw-Hill, New York, NY, USA, 3rd edition, 1987. 


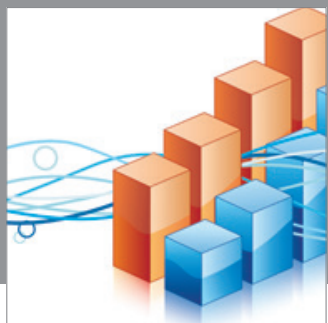

Advances in

Operations Research

mansans

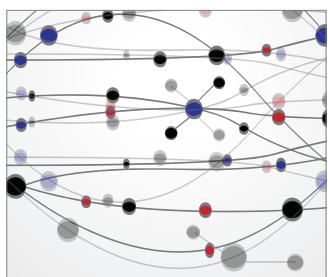

The Scientific World Journal
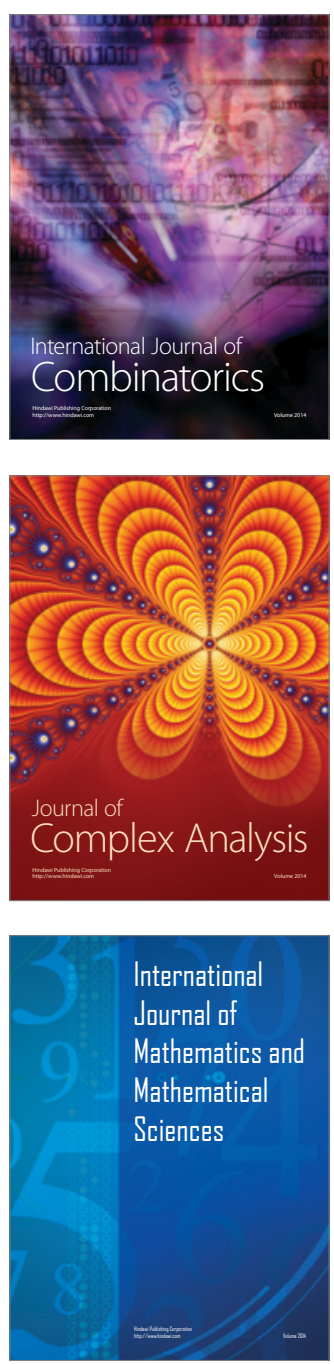
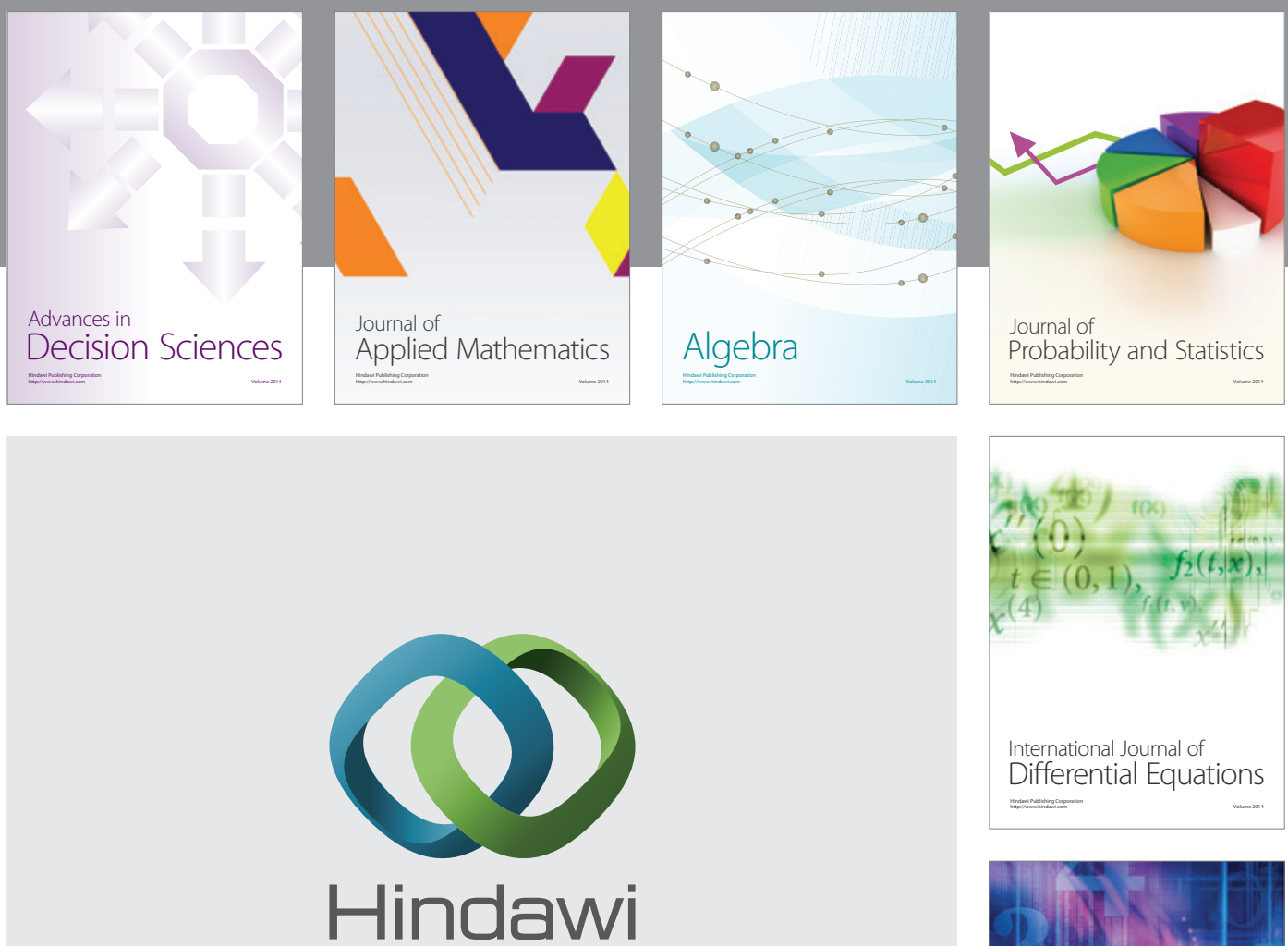

Submit your manuscripts at http://www.hindawi.com
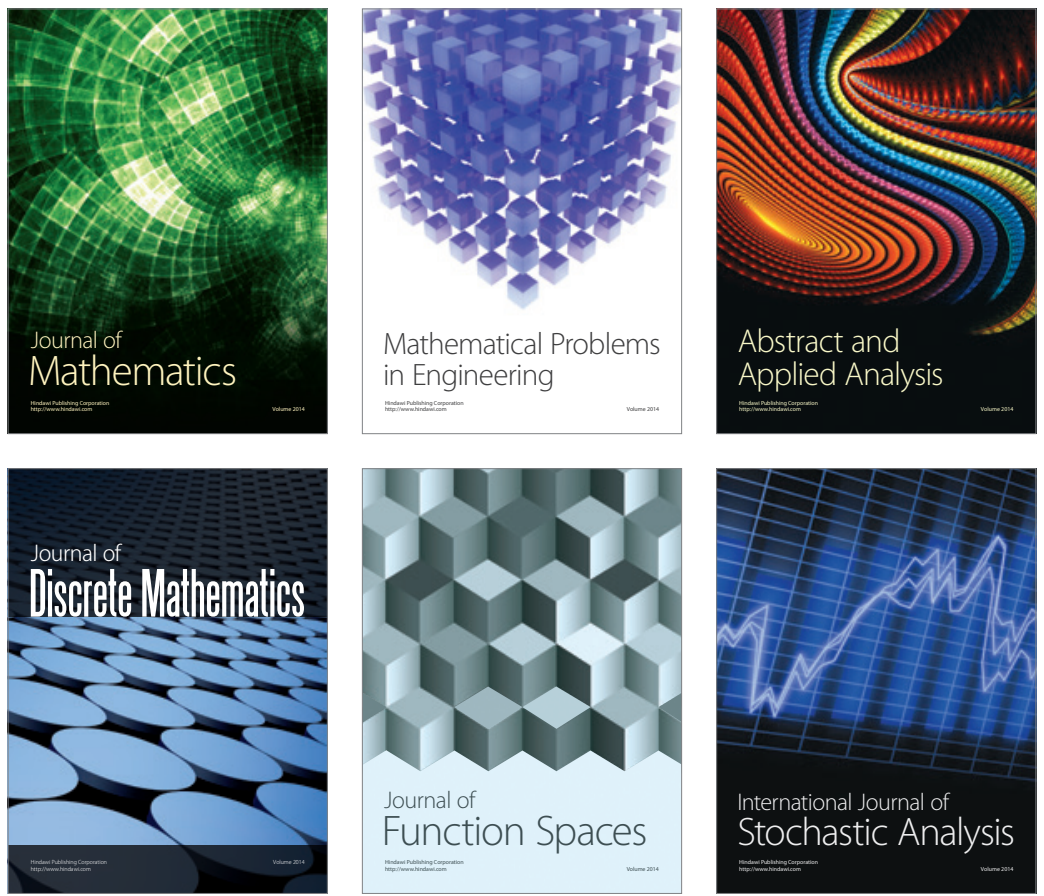

Journal of

Function Spaces

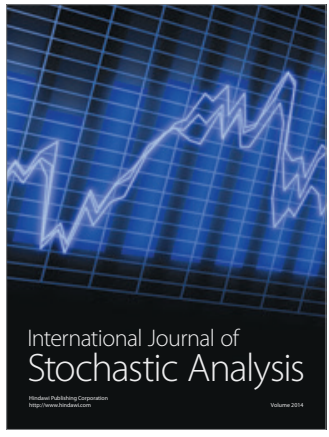

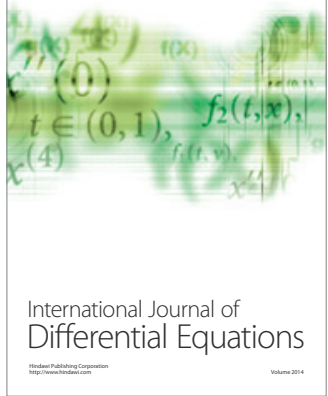
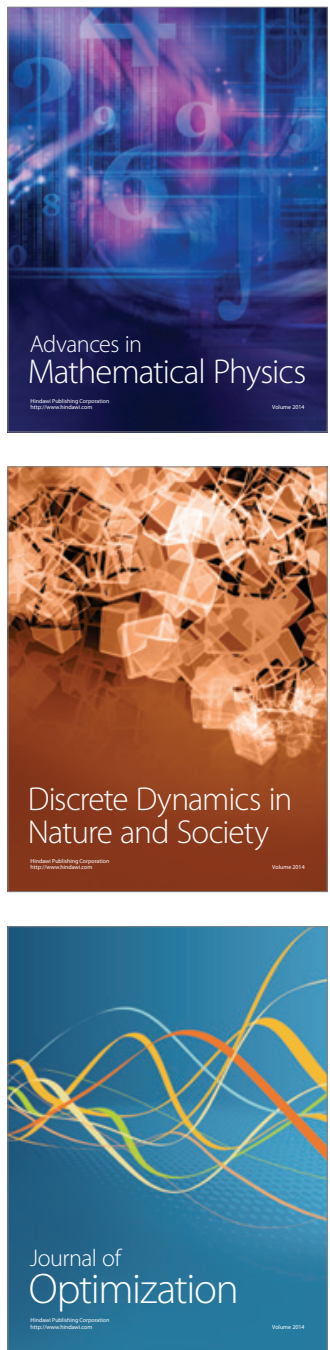